\title{
Exceptional Dehn surgery on the minimally twisted five-chain link
}

\author{
Bruno Martelli, Carlo Petronio and Fionntan Roukema
}

\begin{abstract}
We consider in this paper the minimally twisted chain link with five components in the 3 -sphere, and we analyze the Dehn surgeries on it, namely the Dehn fillings on its exterior $M_{5}$. The 3-manifold $M_{5}$ is a nicely symmetric hyperbolic one, filling which one gets a wealth of hyperbolic 3-manifolds having 4 or fewer (including 0 ) cusps. In view of Thurston's hyperbolic Dehn filling theorem it is then natural to face the problem of classifying all the exceptional fillings on $M_{5}$, namely those yielding non-hyperbolic 3-manifolds. Here we completely solve this problem, also showing that, thanks to the symmetries of $M_{5}$ and of some hyperbolic manifolds resulting from fillings of $M_{5}$, the set of exceptional fillings on $M_{5}$ is described by a very small amount of information.
\end{abstract}

\section{Introduction}

In this paper we establish the following main result. The terminology, the context, the relevance and a conceptual outline of the methods underlying the proof are explained in the next few pages:

Theorem 0.1. Consider the link shown in figure 1, fix a cyclic ordering on its components and employ the meridian-longitude homology bases to parameterize the Dehn surgeries on the link. Then a Dehn surgery is exceptional if and only if up to a composition of the following maps:

$$
\begin{aligned}
&\left(\alpha_{1}, \alpha_{2}, \alpha_{3}, \alpha_{4}, \alpha_{5}\right) \longmapsto\left(\alpha_{5}, \alpha_{1}, \alpha_{2}, \alpha_{3}, \alpha_{4}\right) \\
&\left(\alpha_{1}, \alpha_{2}, \alpha_{3}, \alpha_{4}, \alpha_{5}\right) \longmapsto\left(\alpha_{5}, \alpha_{4}, \alpha_{3}, \alpha_{2}, \alpha_{1}\right), \\
&\left(\alpha_{1}, \alpha_{2}, \alpha_{3}, \alpha_{4}, \alpha_{5}\right) \longmapsto\left(\frac{1}{\alpha_{2}}, \frac{1}{\alpha_{1}}, 1-\alpha_{3}, \frac{\alpha_{4}}{\alpha_{4}-1}, 1-\alpha_{5}\right),
\end{aligned}
$$




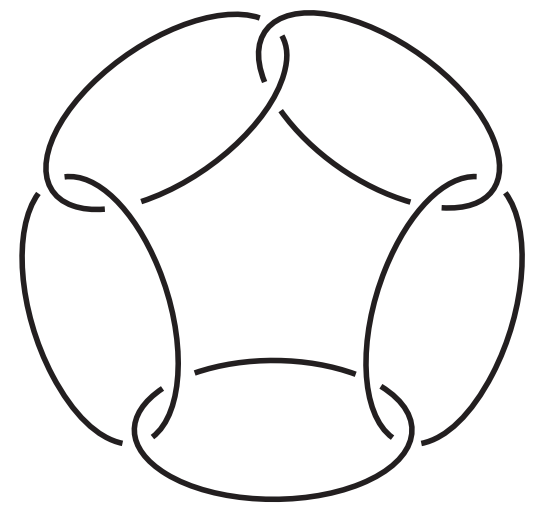

Figure 1: The minimally twisted chain link with five components L10n113.

$$
\begin{aligned}
& \left(-1, \alpha_{2}, \alpha_{3}, \alpha_{4}, \alpha_{5}\right) \longmapsto\left(-1, \alpha_{3}-1, \alpha_{4}, \alpha_{5}+1, \alpha_{2}\right), \\
& (-1,-2,-2,-2, \alpha) \longmapsto(-1,-2,-2,-2,-\alpha-6),
\end{aligned}
$$

it contains one of the next two or it is one of the subsequent five:

$$
\begin{array}{ccc}
\infty \quad(-1,-2,-2,-1) & \left(-2,-\frac{1}{2}, 3,3,-\frac{1}{2}\right) & (-1,-2,-2,-3,-5) \\
(-1,-2,-3,-2,-4) & (-1,-3,-2,-2,-3) & (-2,-2,-2,-2,-2) .
\end{array}
$$

Moreover, no two of these seven fillings are related to each other by any composition of the above five maps.

\subsection{Dehn surgery and Dehn filling}

The operation of Dehn surgery on a link in the 3-sphere, and its natural generalization, termed Dehn filling, are fundamental ones in threedimensional geometric topology. The input of an operation of Dehn filling is given by

- a compact 3-manifold $M$ (that we will always tacitly assume to be orientable and connected) with boundary $\partial M$ consisting of tori and

- a slope (namely, the isotopy class of a non-trivial simple closed unoriented curve) on each component of $\partial M$.

The result of the operation is the manifold obtained by attaching to $M$ a copy of the solid torus $D^{2} \times S^{1}$ along each component of $\partial M$, with a solid 
torus attached to a component $T$ of $\partial M$ so that the meridian $\left(\partial D^{2}\right) \times\{*\}$ is matched to the slope $\alpha$ contained in $T$. To allow $T$ to be left unfilled, one also allows $\alpha$ to be the empty slope. A Dehn surgery on a link $L$ in the 3 -sphere is a Dehn filling on its exterior (the complement of an open regular neighborhood). To highlight the importance of this operation we recall for instance the celebrated theorem of Lickorish [28], according to which every closed (orientable) 3-manifold is the result of a Dehn surgery on some link in $S^{3}$. In the rest of this paper, when a manifold $M$ bounded by tori is given, by a (Dehn) filling on $M$ we will mostly refer to the set of slopes along which the filling has to be performed. Occasionally, we will also use the same term to refer to the manifold resulting from the operation of filling along the given slopes, but when there is any risk of confusion we will distinguish between the filling (viewed as an instruction) and its result.

\subsection{Hyperbolic manifolds and exceptional fillings}

We say that $M$ is hyperbolic [4] if its interior admits a complete hyperbolic metric with finite volume, in which case the components of $\partial M$ correspond to the cusps of the interior of $M$. The famous hyperbolic Dehn filling theorem of Thurston [29] states that if $M$ is hyperbolic then "most" Dehn fillings on $M$ give manifolds that are also hyperbolic. A Dehn filling not producing a hyperbolic manifold is called exceptional. We continue here the program initiated in [24] of classifying the exceptional fillings on hyperbolic manifolds with an increasing number of boundary components. Namely, we provide a complete classification of all the exceptional fillings on the exterior $M_{5}$ of the minimally twisted chain link with five components in the 3-sphere, shown in figure 1 and denoted by L10n113 in Thistlewaite's tables.

The relevance of the 5-cusped hyperbolic manifold $M_{5}$ comes from the following facts. First, it is conjecturally [2] the 5-cusped manifold with smallest volume $10.149 \ldots$ and smallest Matveev complexity 10 (it can be triangulated using 10 regular ideal hyperbolic tetrahedra, see below). Second, by filling $M_{5}$ one obtains a multitude of interesting manifolds, including most manifolds from the cusped census [6], and many 1-cusped manifolds having interesting exceptional fillings, such as various families of Berge [3] knot exteriors, all Eudave-Muñoz [12,13] knot exteriors, and all exceptional reducible-toroidal pairs at maximal distance [21]. Third, the manifold $M_{5}$ has a nice and unusually large symmetry group, because it double covers a very natural and symmetric orbifold, called the pentangle, with underlying 
space $S^{3}$ viewed as the boundary of the 4-simplex and as singular set the 1-skeleton of the 4-simplex.

The exceptional fillings on $M_{5}$ are classified by Theorem 0.1 . Our proof of this result was computer-assisted but rigorous, namely immune from drawbacks coming from numerical approximation. We used a python code named find_exceptional_fillings.py, written by the first author and publicly available from [23]. The code uses the $S n a p P y$ libraries [9], takes as an input any hyperbolic manifold $M$ (with an arbitrary number of cusps), and gives as an output a list of candidate exceptional fillings on $M$, including all truly exceptional ones. The fact that indeed all truly exceptional fillings are included follows from the use of the hikmot library [15], that allows one to make SnapPy's numerical calculations rigorous. For $M_{5}$, the list of candidate exceptional fillings actually turned out to be extremely small up to the action of some symmetry groups, and the conclusion of the proof of Theorem 0.1 was then obtained by explicitly recognizing the manifolds resulting from the candidate exceptional fillings, thus proving them not to be hyperbolic; this process was carried out both by hand and using the Matveev-Tarkaev [25] nice Recognizer program. We point out that the code find_exceptional_fillings.py can be used on any hyperbolic manifold. We have tested the code on various manifolds and found that the output list of candidate exceptional fillings is typically correct (i.e., all the fillings in the list are indeed exceptional). The proof of Theorem 0.1 contained in Section 3 below may be viewed as a tutorial introduction to the code.

One of the key features of Theorem 0.1 is in our opinion the unexpected shortness of its statement. In fact, our results show that very little information is needed to list all the exceptional fillings on $M_{5}$, and also to give a very precise description of all the corresponding filled manifolds. This is due to the many symmetries possessed by $M_{5}$ and by some hyperbolic manifolds obtained as Dehn fillings of $M_{5}$. In fact, Theorem 0.1 shows that only seven exceptional fillings on $M_{5}$ are responsible for all the other ones and the geometric meaning of the maps appearing in the statement will be made more precise soon. In addition, a single exceptional filling on $M_{5}$ the first one listed in Theorem 0.1 - is responsible for the vast majority of the other ones: the remaining six exceptional fillings can be viewed as being very sporadic. Since most of the manifolds in the Callahan et al. [6] cusped census can be obtained as fillings of $M_{5}$, we can conclude that most of the exceptional fillings on the manifolds in the census are determined by this single exceptional filling on $M_{5}$. For instance, all the 10 exceptional surgeries on the figure-eight knot are consequences of it. 

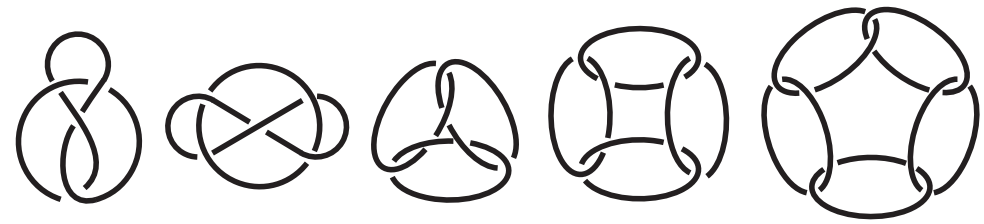

Figure 2: The links in $S^{3}$ whose exteriors are denoted by $M_{1}, M_{2}, M_{3}, M_{4}$ and $M_{5}$.

\subsection{A notable finite(?) sequence of cusped hyperbolic manifolds}

Figure 2 shows five links in the 3 -sphere, at least the first three of which are famous ones: the figure-eight knot $\mathrm{K} 4 \mathrm{a} 1=4_{1}$, the Whitehead link $\mathrm{L} 5 \mathrm{a} 1=5_{1}^{2}$ and the chain link with three components $\mathrm{L} 6 \mathrm{a} 5=6_{1}^{3}$; we then have the chain $\mathrm{L} 8 \mathrm{n} 7=8_{2}^{4}$ link with four components, and (of course) the minimally twisted 5-chain link L10n113 (for the first four links we are indicating both Thistlewaite's and Rolfsen's names). Let us now denote by $M_{i}$ the exterior of the $i$ th link in figure 2 (the notation is consistent for $M_{5}$ ). It is well known that each $M_{i}$ is an $i$-cusped hyperbolic manifold, and the finite sequence $\left(M_{i}\right)_{i=1}^{5}$ has several interesting features. We first note that $M_{i}$ is a filling of $M_{i+1}$ for all $i \leqslant 4$. We then recall that each $M_{i}$ is conjectured [2] to have the smallest volume among $i$-cusped hyperbolic manifolds; the conjecture was proved in [7] for $i=1$ and in [2] for $i=2$, and it is open for $i=3,4,5$. But the most remarkable properties of $\left(M_{i}\right)_{i=1}^{5}$ arise when one considers their exceptional fillings. The manifold $M_{3}$ was already called the magic one in $[13,14]$, because it has many notable exceptional fillings. The exceptional fillings on $M_{1}$ were classified by Thurston [29], those on $M_{2}$ and $M_{3}$ were classified by Martelli and Petronio [24] and those on $M_{4}$ and $M_{5}$ are classified here.

Our next aim is to explain our discovery that the amount of information required to describe the exceptional fillings on $M_{i}$ is roughly constant for $i=1,2,3,4,5$. Considering that the number of cusps and the volume of $M_{i}$ increase with $i$, we believe that this is a rather remarkable fact. However, to substantiate our statement we need to be a little more specific. In fact, as soon as a hyperbolic manifold $M$ has more than one cusp, infinitely many fillings on $M$ are typically exceptional, but finitely many fillings are responsible for all other ones. To make this explicit, we define an exceptional filling on $M$ to be isolated if all its proper sub-fillings (namely, those obtained by replacing at least one non-empty slope with an empty one) are hyperbolic. A filling having an exceptional proper sub-filling is exceptional unless a very 
Table 1: Number of isolated exceptional fillings on $M_{i}$ according to the number of filled cusps.

\begin{tabular}{lcccccr}
\hline & \multicolumn{7}{c}{ Number of cusps filled } & \\
\cline { 2 - 6 } Manifold & 1 & 2 & 3 & 4 & 5 & Total \\
\hline$M_{1}$ & 10 & & & & & 10 \\
$M_{2}$ & 12 & 14 & & & & 26 \\
$M_{3}$ & 15 & 15 & 52 & & & 82 \\
$M_{4}$ & 16 & 24 & 96 & 492 & & 628 \\
$M_{5}$ & 15 & 30 & 180 & 780 & 5232 & 6237 \\
\hline
\end{tabular}

Table 2: Number of isolated exceptional fillings on $M_{i}$ according to the number of filled cusps, after identifying fillings obtained from each other under the action of the symmetry group of $M_{i}$.

\begin{tabular}{lrrrrrr}
\hline & \multicolumn{5}{c}{ Number of cusps filled } & \\
\cline { 2 - 5 } Manifold & 1 & 2 & 3 & 4 & 5 & Total \\
\hline$M_{1}$ & 6 & & & & & 6 \\
$M_{2}$ & 6 & 8 & & & & 14 \\
$M_{3}$ & 5 & 3 & 14 & & & 22 \\
$M_{4}$ & 2 & 2 & 4 & 22 & & 30 \\
$M_{5}$ & 1 & 1 & 3 & 7 & 52 & 64 \\
\hline
\end{tabular}

special situation occurs, which is never the case for our $M_{i}$ 's. We can then conclude that a filling on $M_{i}$ is exceptional if and only if it contains an isolated exceptional filling.

Getting to the actual data we have obtained, we start by listing in table 1 the numbers of isolated exceptional fillings on $M_{i}$ for $i=1,2,3,4,5$, split according to the number $k$ of cusps filled. As one sees, these numbers grow with $i$ and $k$, and the total number of isolated exceptional fillings of $M_{i}$ appears to grow exponentially with $i$. These numbers already reduce very considerably if we take into account the action of the symmetry group of $M_{i}$, identifying exceptional fillings equivalent under it, as shown in table 2 .

But the dramatic conclusion that the number of "really inequivalent" exceptional fillings of $M_{i}$ is roughly constant for $i=1,2,3,4,5$ follows by taking into account another phenomenon. In fact, it can and does happen that a hyperbolic manifold $N$ obtained by filling some $M_{i}$ has symmetries that are not induced by symmetries of $M_{i}$. If this situation, two isolated exceptional fillings on $N$ that are equivalent under such symmetries of $N$ 
Exceptional Dehn surgery on the minimally twisted five-chain link 695

Table 3: Number of isolated exceptional fillings on $M_{i}$ according to the number of filled cusps, after identifying fillings obtained from each other under the action of the symmetry group of $M_{i}$ or of hyperbolic manifolds obtained by filling $M_{i}$.

\begin{tabular}{lcccccc}
\hline & \multicolumn{5}{c}{ Number of cusps filled } & \\
\cline { 2 - 5 } Manifold & 1 & 2 & 3 & 4 & 5 & Total \\
\hline$M_{1}$ & 6 & & & & & 6 \\
$M_{2}$ & 6 & 2 & & & & 8 \\
$M_{3}$ & 5 & 1 & 3 & & & 9 \\
$M_{4}$ & 2 & 0 & 1 & 3 & & 6 \\
$M_{5}$ & 1 & 0 & 0 & 1 & 5 & 7 \\
\hline
\end{tabular}

Table 4: Computer time needed to classify the exceptional fillings.

\begin{tabular}{lcccc}
\hline Manifold & $M_{2}$ & $M_{3}$ & $M_{4}$ & $M_{5}$ \\
\hline Computer time & $1^{\prime \prime}$ & $24^{\prime \prime}$ & $2^{\prime} 10^{\prime \prime}$ & $3^{\prime} 48^{\prime \prime}$ \\
\hline
\end{tabular}

both contribute to the counting in table 2 , but after all we can still identify them, letting also the symmetries of $N$ act. Similarly, there can be an isolated exceptional filling on $N$ that contributes to table 2 but is, under symmetries of $N$, equivalent to a non-isolated filling on $M_{i}$, in which case we can disregard it. By systematically taking into account the symmetries of the hyperbolic fillings of the $M_{i}$ 's we then get the figures of table 3 (with the seven fillings on $M_{5}$ being precisely those described in Theorem 0.1). This proves that the minimal number of exceptional fillings needed to generate (via symmetries) all the exceptional fillings on $M_{1}, M_{2}, M_{3}, M_{4}$ and $M_{5}$ is indeed roughly constant.

We underline now that not only is the number of exceptional fillings on $M_{i}$ for $i=1,2,3,4,5$ responsible for all other exceptional fillings extremely small, but also that, once the investigation is properly organized, the computer time needed to detect them is very limited as well, see table 4. Encouraged by these facts, we believe that it should be possible to carry out a similar analysis for manifolds having six or more cusps, and we put forward two questions. To state them, we define a sequence $\left(M_{i}\right)_{i=1}^{+\infty}$ to be universal if every compact 3-manifold with (possibly empty) boundary consisting of tori can be obtained by Dehn filling on some $M_{i}$. We then have the following (slightly vague): 
Question 0.2. Is there a universal sequence $\left(M_{i}\right)_{i=1}^{+\infty}$ with $M_{i}$ an $i$-cusped hyperbolic 3-manifold such that the exceptional fillings on each $M_{i}$ can be described using a small amount of data?

A much more ambitious question is the following:

Question 0.3. Is there a universal sequence $\left(M_{i}\right)_{i=1}^{+\infty}$ with $M_{i}$ an $i$-cusped hyperbolic 3-manifold such that the exceptional fillings on all the $M_{i}$ 's can be simultaneously described using a finite amount of data?

\subsection{On the computer assisted-proof}

In the original 2011 version of this paper, to rigorously verify the numerical computations made by SnapPy, we used an algorithm constructed in $[26,27]$ and implemented in Pari [8]. It was then pointed out to us that this algorithm might not be $100 \%$ immune from round-off errors. Motivated by another project [17], Hoffman et al. [15] wrote in 2013 a different algorithm that has at least three advantages: it uses interval arithmetic (and is hence immune from round-off errors), it is very fast, and it is written directly in python (so we were able to incorporate it directly in our previous code). The 2013 versions of our paper and codes use this new algorithm.

\section{Main results}

We describe here in greater detail the hyperbolic manifold $M_{5}$, namely the exterior of the minimally twisted chain link with five components L10n113 shown in figure 1. In particular, we list its symmetries and we analyze some of its notable exceptional and hyperbolic fillings. We then state Theorem 1.2, that classifies all the exceptional fillings on $M_{5}$.

\subsection{Hyperbolic structure}

The reflection (or rotation of angle $\pi$ ) across the dotted circle in figure 3 (left) leaves the link invariant and thus gives an involution $\iota$ on $M_{5}$. The quotient of $M_{5}$ under $\iota$ is an orbifold whose singular set consists of 10 order2 edges, bounded by 5 spheres with 4 order- 2 cone points, each of which is the quotient of a toric component of $\partial M_{5}$ under the elliptic involution. Collapsing these spheres to points we get an orbifold with total space $S^{3}$ and singular set the pentangle graph shown in figure 3 (right). This implies 

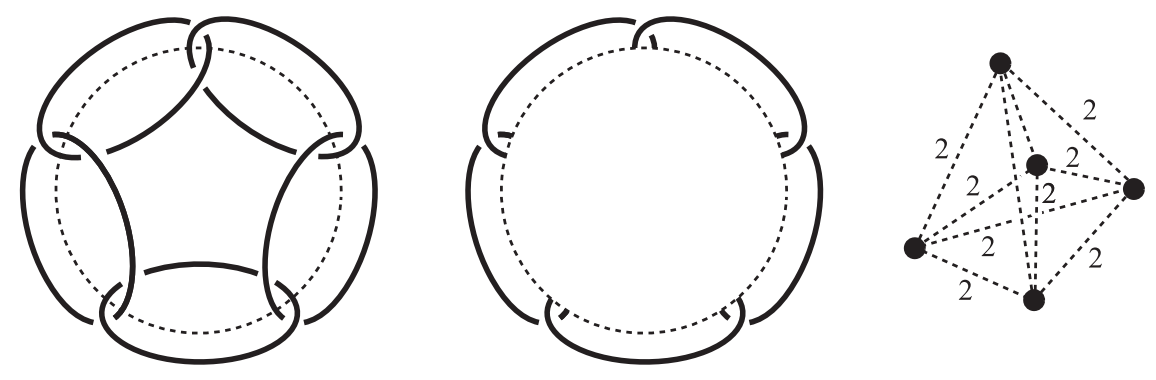

Figure 3: Left: a circle the reflection across which leaves the link invariant. Center: the corresponding quotient graph. Right: the pentangle graph, obtained from the quotient graph by contracting each solid arc to a vertex.

that the interior of $M_{5}$ is the double branched cover of $S^{3}$ minus the vertices of the pentangle, branched along the edges of the pentangle.

We will now construct the hyperbolic structure on (the interior of) $M_{5}$ as the double cover of the hyperbolic structure on the pentangle orbifold (minus the vertices), as described in [10]. To this end, consider $S^{3}$ to be the boundary of the four-dimensional simplex and the pentangle graph to be the 1 -skeleton of this 4-simplex. Next, realize each of the 5 codimension 1 -faces of the 4-simplex as a regular ideal hyperbolic tetrahedron in $\mathbb{H}^{3}$, and glue the faces of these ideal tetrahedra using isometries. Each ideal tetrahedron has dihedral angle $\frac{\pi}{3}$ along each of its edges, and every edge of the pentangle graph is adjacent to three ideal tetrahedra, so the cone angle along each edge of the pentangle graph is $3 \times \frac{\pi}{3}=\pi$. The link of each vertex of the pentangle consists of four Euclidean equilateral triangles that glue nicely to give a Euclidean structure with four cone points of angle $\pi$ on the 2-sphere, so we get an orbifold hyperbolic structure on the pentangle graph. Pulling back this structure to $M_{5}$ we see that its hyperbolic structure is obtained by gluing together 10 regular ideal hyperbolic tetrahedra. In particular, the volume of $M_{5}$ is equal to $10 \times v_{3}=10.149416 \ldots$

\subsection{Symmetries and slopes}

Every permutation of the vertices of the pentangle graph is realized by a unique isometry of the pentangle orbifold, whose symmetry group is therefore $\mathfrak{S}_{5}$. Every isometry of the quotient lifts to $M_{5}$, whose symmetry group is seen to be isomorphic to $\mathfrak{S}_{5} \times \mathbb{Z} / 2$, with the factor $\mathbb{Z} / 2$ generated by the involution $\iota$ and $\mathfrak{S}_{5}$ permuting the cusps, as one can check using SnapPy [9]. Note that the symmetry group of $M_{5}$ is larger than the symmetry group of 
L10n113: the latter group is isomorphic to $D_{5} \times \mathbb{Z} / 2$, with $\mathbb{Z} / 2$ again generated by $\iota$ and $D_{5}$ being the order-10 dihedral group, generated by a rotation of angle $\frac{2 \pi}{5}$ around an axis orthogonal to the projection plane in figure 1 , and by the reflection across a suitable plane containing this axis.

Let us now number from 1 to 5 the components of L10n113, in such a way that the $i$ th component is linked with the $(i+1)$ th (there is a $D_{5}$ ambiguity for doing this, that we will soon view to be immaterial). Correspondingly, we have a numbering from 1 to 5 of the components of $\partial M_{5}$. We also fix a meridian-longitude oriented homology basis $\left(\mu_{i}, \lambda_{i}\right)$ on the $i$ th component of $\partial M_{5}$, viewed as the boundary of the exterior of a (trivial) knot in $S^{3}$. This basis is defined up to simultaneous sign reversal, so if a slope represents $\pm\left(p_{i} \mu_{i}+q_{i} \lambda_{i}\right)$ in homology we have an element $p_{i} / q_{i}$ of $\mathbb{Q} \cup\{\infty\}$ uniquely defined by the slope, and conversely. This shows that a filling on one boundary component is described by an element of $\Phi=\mathbb{Q} \cup\{\infty, \emptyset\}$. Note that $\infty$ is the meridian and 0 is the longitude. Using the numbering we then see that a filling on $M_{5}$ is described by a 5 -tuple $\left(\alpha_{1}, \alpha_{2}, \alpha_{3}, \alpha_{4}, \alpha_{5}\right) \in \Phi^{5}$. The corresponding filled manifold will be denoted by $M_{5}\left(\alpha_{1}, \alpha_{2}, \alpha_{3}, \alpha_{4}, \alpha_{5}\right)$.

Every symmetry of $M_{5}$ sends a slope on a component of $\partial M_{5}$ to some (other) slope on some (other) component of $\partial M_{5}$, so we have an action of $\mathfrak{S}_{5}$ on $\Phi^{5}$ (one easily sees that $\iota$ acts trivially on all slopes, so we dismiss it). To describe this action we start with the easy part coming from the (dihedral) symmetries of the link: each such symmetry send meridians to meridians and longitudes to longitudes, therefore the action of $D_{5}$ on $\Phi^{5}$ is generated by the maps

$$
\begin{aligned}
& \left(\alpha_{1}, \alpha_{2}, \alpha_{3}, \alpha_{4}, \alpha_{5}\right) \longmapsto\left(\alpha_{5}, \alpha_{1}, \alpha_{2}, \alpha_{3}, \alpha_{4}\right), \\
& \left(\alpha_{1}, \alpha_{2}, \alpha_{3}, \alpha_{4}, \alpha_{5}\right) \longmapsto\left(\alpha_{5}, \alpha_{4}, \alpha_{3}, \alpha_{2}, \alpha_{1}\right) .
\end{aligned}
$$

This implies in particular that the above-chosen ordering of the components of $\partial M$ is irrelevant. To generate the full action of $\mathfrak{S}_{5}$ on $\Phi^{5}$ it is now sufficient to add the action on slopes of a symmetry that switches two boundary components leaving the other three invariant. Using SnapPy one sees that one such map is as follows:

$$
\left(\alpha_{1}, \alpha_{2}, \alpha_{3}, \alpha_{4}, \alpha_{5}\right) \longmapsto\left(\frac{1}{\alpha_{2}}, \frac{1}{\alpha_{1}}, 1-\alpha_{3}, \frac{\alpha_{4}}{\alpha_{4}-1}, 1-\alpha_{5}\right) .
$$

This is an orientation-reversing isometry. Note that the action on slopes on the invariant components is a non-trivial one; moreover, any of the slopes in the argument of the map is allowed to be $\emptyset$, in which case there is a corresponding $\emptyset$ slope in the value. 
Exceptional Dehn surgery on the minimally twisted five-chain link 699

\subsection{Notation for some graph manifolds}

Let $\Sigma$ be an oriented surface, possibly with boundary. Given $k \in \mathbb{N}$ and coprime pairs of integers $\left(p_{i}, q_{i}\right)$ for $i=1, \ldots, k$ with $p_{i} \neq 0$ for all $i$, we denote by

$$
\left(\Sigma,\left(p_{1}, q_{1}\right), \ldots,\left(p_{k}, q_{k}\right)\right)
$$

the Dehn filled manifold $\left(\Sigma^{\prime} \times S^{1}\right)\left(p_{1} \mu_{1}+q_{1} \lambda_{1}, \ldots, p_{k} \mu_{k}+q_{k} \lambda_{k}\right)$, where $\Sigma^{\prime}$ is $\Sigma$ with $k$ open discs removed with the induced orientation, $\mu_{i}$ is the oriented component of $\partial \Sigma^{\prime}$ corresponding to the $i$ th disc removed, and $\lambda_{i}$ is the oriented $S^{1}$ on the same torus. The result is a Seifert manifold with an exceptional fiber for each $i$ such that $\left|p_{i}\right| \geqslant 2$. As an example of this construction we note that the Poincaré homology sphere can be described as

$$
\left(S^{2},(2,-1),(3,1),(5,1)\right) .
$$

If we allow one $p_{i}$ to be 0 , say $p_{1}=0$ and $q_{1}=1$, then $\left(\Sigma,\left(p_{1}, q_{1}\right), \ldots,\left(p_{k}, q_{k}\right)\right)$ turns out to be the connected sum of the lens spaces $L\left(p_{2}, q_{2}\right), \ldots, L\left(p_{k}, q_{k}\right)$ and of $2 g$ copies of $S^{2} \times S^{1}$ if the genus of $\Sigma$ is $g$, with $h$ unlinked unknots removed if $\partial \Sigma$ has $h$ components.

Our notation to encode (some) Seifert manifolds can now be promoted to encode (some) graph manifolds. In fact, note that the boundary of $\left(\Sigma,\left(p_{1}, q_{1}\right)\right.$, $\left.\ldots,\left(p_{k}, q_{k}\right)\right)$ is given by $(\partial \Sigma) \times S^{1}$, so it consists of tori, and that on each of them there is a preferred homology basis given by an oriented component of $\partial \Sigma$ and the oriented $S^{1}$. Moreover, any two boundary components are mapped to each other by a homeomorphism of the manifold that matches the homology bases. Given two such manifolds $M$ and $M^{\prime}$ and a matrix $X \in$ $\mathrm{GL}(2, \mathbb{Z})$ we can therefore define without ambiguity the gluing $M \bigcup_{X} M^{\prime}$, along a homeomorphism from a boundary component of $M$ to one of $M^{\prime}$ whose action on homology is expressed by $X$ with respect to the given bases. Note that $M$ and $M^{\prime}$ are oriented, so $M \bigcup_{X} M^{\prime}$ is naturally oriented if $\operatorname{det}(X)=-1$, while it is merely orientable if $\operatorname{det}(X)=+1$. But we can always reverse the orientation of $M$, which corresponds to changing each $\left(p_{i}, q_{i}\right)$ to $\left(p_{i},-q_{i}\right)$, to get $\operatorname{det}(X)=-1$, that we will always do for aesthetic reasons even if we only care about orientable but unoriented manifolds. In a similar way one can define a manifold $M / X$ by gluing together along $X \in \mathrm{GL}(2, \mathbb{Z})$ two boundary components of the same $M$, but in this case one must have $\operatorname{det}(X)=-1$ to get an orientable result. Denoting by $D$ and $A$ the disc and the annulus, respectively, we can consider for instance the 


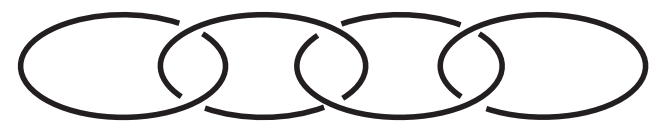

Figure 4: An $\infty$ filling gives the exterior of an open chain with four components (the connected sum of three copies of the Hopf link L2a1 $=2_{1}^{2}$ ).

following closed graph manifolds:

$$
(D,(2,1),(2,-1)) \bigcup_{\left(\begin{array}{ll}
1 & 5 \\
1 & 4
\end{array}\right)}(D,(2,1),(3,2)), \quad(A,(2,1)) /_{\left(\begin{array}{ll}
1 & 2 \\
1 & 1
\end{array}\right)} .
$$

Note that if a gluing of two Seifert manifolds (or a gluing of a Seifert manifold to itself) is performed along $X \in \mathrm{GL}(2, \mathbb{Z})$, then the absolute value of the top-right entry in $X$ has an instrinsic meaning, since it represents the geometric intersection number on the gluing torus between the two Seifert fibers coming from opposite sides of the torus.

\subsection{Some notable exceptional fillings}

We describe here some exceptional fillings on $M_{5}$. Theorem 1.2 will then assert that these are the only ones up to the maps (1.1) to (1.3) induced by the symmetries of $M_{5}$ and up to further maps described below coming from symmetries of hyperbolic fillings of $M_{5}$. In the sequel for $k<5$ and $\alpha_{1}, \ldots, \alpha_{k} \in \Phi \backslash\{\emptyset\}$ we will interpret $\left(\alpha_{1}, \ldots, \alpha_{k}\right)$ as the filling $\left(\alpha_{1}, \ldots, \alpha_{k}\right.$, $\emptyset, \ldots, \emptyset) \in \Phi^{5}$ on $M_{5}$.

To begin, we note that filling the exterior of a link with a slope $\infty$ corresponds to canceling the link component corresponding to the filled boundary component of the exterior. Therefore $M_{5}(\infty)$ is the exterior of the open chain link with four components shown in figure 4 ; denoting by $P$ the pair-of-pants one then easily sees that $M_{5}(\infty)$ is the graph manifold

$$
F=\left(P \times S^{1}\right) \bigcup_{\left(\begin{array}{ll}
0 & 1 \\
1 & 0
\end{array}\right)}\left(P \times S^{1}\right)
$$

Applying the map (1.3) with $\alpha_{1}=\infty$ and the other $\alpha_{i}$ 's empty, and then with $\alpha_{4}=\infty$ and the other $\alpha_{i}$ 's empty, we see that the slopes 0 and 1 are equivalent to $\infty$ up to the symmetries of $M_{5}$, therefore $M_{5}(0)=M_{5}(1)=F$. More exactly we already have three exceptional slopes on each component of $\partial M_{5}$, for a total of 15 , giving $F$ as a filling, and one easily sees that no other slope is obtained from them under the maps (1.1) to (1.3). 
Exceptional Dehn surgery on the minimally twisted five-chain link 701

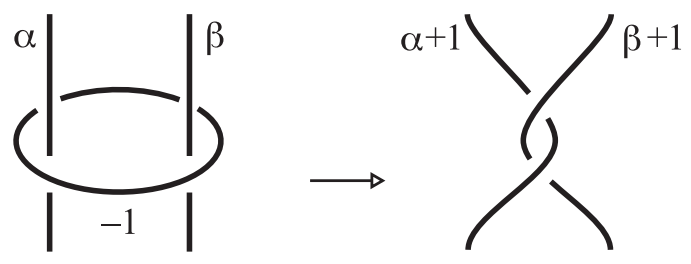

Figure 5: The blow-down: a Fenn-Rourke move on surgery diagrams. On the left, the two strands piercing the spanning disc of the unknot are supposed to be parts of two distinct components of the link.

Remark 1.1. If $\alpha \in \Phi^{5}$ contains some slope 0,1 or $\infty$, then $M_{5}(\alpha)$ is a filling of $F$, and a considerable variety of different filled manifolds can already be obtained. The closed ones, for instance, have the form

$$
(D,(a, b),(c, d)) \bigcup_{\left(\begin{array}{ll}
0 & 1 \\
1 & 0
\end{array}\right)}(D,(e, f),(g, h))
$$

for arbitrary filling coefficients $a, \ldots, h$. If $|a|,|c|,|e|,|g|$ are all at least 2 then this is an irreducible 3-manifold whose Jaco-Shalen-Johannson (JSJ) decomposition $[18,19]$ consists of two blocks and is transparent from the notation. Allowing some of $a, c, e, g$ to be 0 or \pm 1 we also get all small Seifert spaces and all reducible manifolds of the form $L(p, q) \# L(r, s)$.

As we will see, the single exceptional filling $\infty$ is responsible for the vast majority of the exceptional fillings on $M_{5}$. There are however a few sporadic cases of an independent nature, that we will now describe, to do which we will first study some notable hyperbolic fillings of $M_{5}$, starting from $M_{5}(-1)$. To understand $M_{5}(-1)$ and its fillings we recall that a filling of a link exterior can be described diagrammatically by attaching a symbol in $\Phi$ to each link component. Attaching a -1 to a component of L10n113 and applying the Fenn-Rourke move described in figure 5 (and called blowdown in the sequel) we see that $M_{5}(-1)$ is actually the exterior $M_{4}$ of the 4-chain link L8n7 shown above in figure 2. Using SnapPy one then sees that $M_{4}$ is a hyperbolic manifold obtained by suitably pairing the faces of two regular ideal octahedra in $\mathbb{H}^{3}$, therefore it has volume $2 \times 3.66386238 \ldots=$ $7.32772475 \ldots$... The blow-down of figure 5 also shows that

$$
M_{5}\left(-1, \alpha_{2}, \ldots, \alpha_{5}\right)=M_{4}\left(\alpha_{2}+1, \alpha_{3}, \alpha_{4}, \alpha_{5}+1\right)
$$

after fixing a cyclic ordering of the components of L8n7. More precisely, the slopes on $\partial M_{5}(-1)$ represented by $\alpha_{2}, \alpha_{3}, \alpha_{4}, \alpha_{5} \in \Phi$ using the homology 

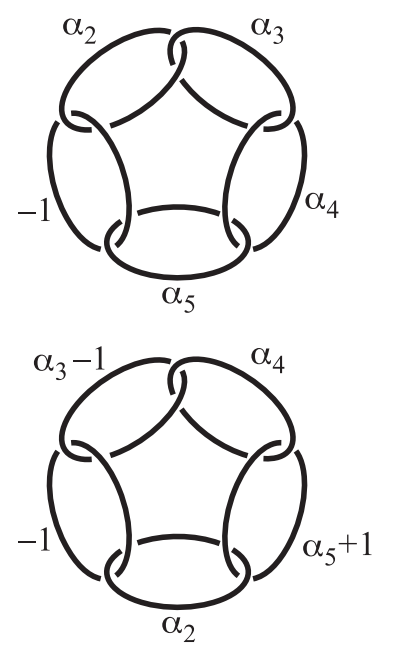
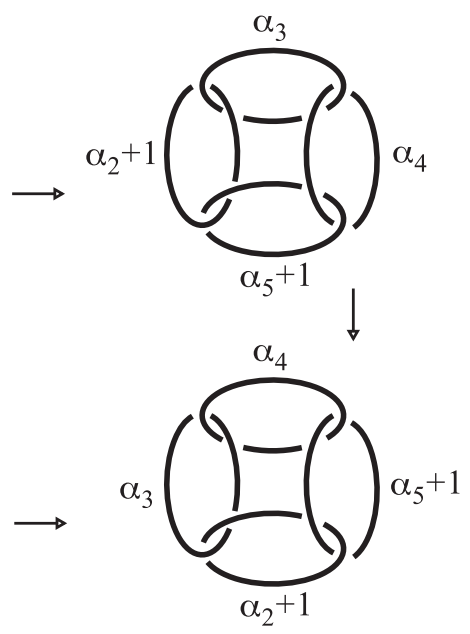

Figure 6: Two distinct sets of slopes on the second to fifth component of $\partial M_{5}$ give on $M_{4}=M_{5}(-1)$ slopes that are obtained from each other by the order-4 symmetry of $M_{4}$.

bases coming from L10n113 are the slopes on $\partial M_{4}$ represented by $\alpha_{2}+$ $1, \alpha_{3}, \alpha_{4}, \alpha_{5}+1 \in \Phi$ using the bases coming from L8n7. The link L8n7 has an obvious order-4 symmetry (a rotation of angle $\frac{\pi}{2}$ around an axis orthogonal to the projection plane) giving a symmetry of $M_{4}$ that is not induced by a symmetry of $M_{5}$, and figure 6 shows how this symmetry can be translated into the map

$$
\left(-1, \alpha_{2}, \alpha_{3}, \alpha_{4}, \alpha_{5}\right) \longmapsto\left(-1, \alpha_{3}-1, \alpha_{4}, \alpha_{5}+1, \alpha_{2}\right)
$$

acting on $\{-1\} \times \Phi^{4}$ viewed as a subset of the fillings $\Phi^{5}$ on $M_{5}$. One easily sees that (1.4) indeed cannot be deduced from (1.1) to (1.3). We also note that the latter functions map -1 to $\frac{1}{2}$ or 2 , so 15 different fillings on $M_{5}$ give $M_{4}$.

The rest of the sequence of link exteriors $\left(M_{i}\right)_{i=1}^{5}$ introduced in figure 2 is obtained in a similar fashion, with $M_{i-1}=M_{i}(-1)$ as shown in figure 7 , whence

$$
\begin{array}{ll}
M_{4}=M_{5}(-1), & M_{3}=M_{5}(-1,-2), \\
M_{2}=M_{5}(-1,-2,-2), & M_{1}=M_{5}(-1,-2,-2,-2)
\end{array}
$$

(though several alternative realizations of $M_{i}$ as a filling of $M_{5}$ exist). Of the links in figure 2, only the figure-eight knot is amphichiral, and its equivalence 
Exceptional Dehn surgery on the minimally twisted five-chain link 703
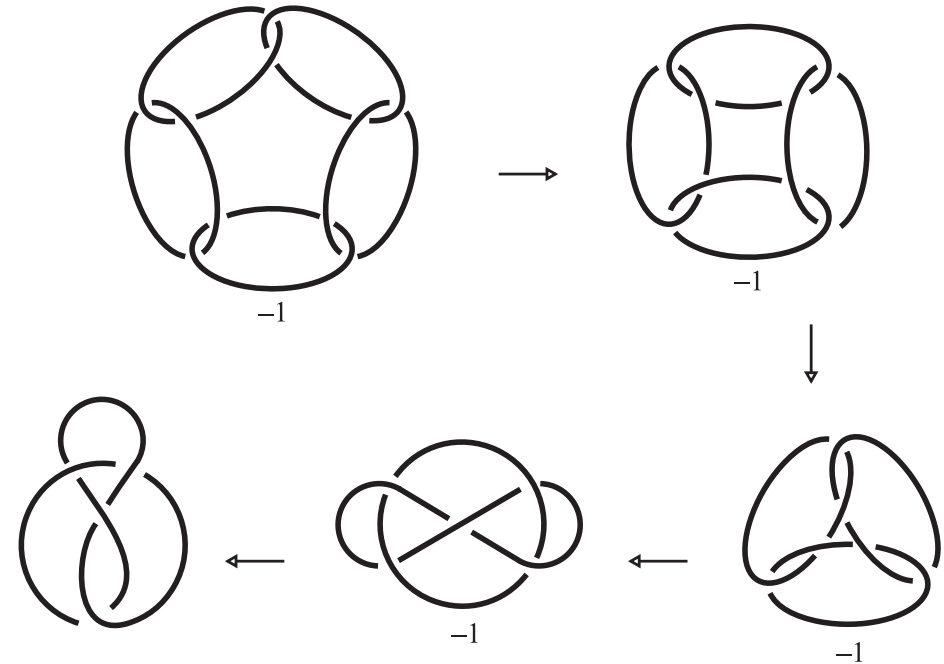

Figure 7: Each $M_{i-1}$ as a (-1)-filling on $M_{i}$ for $i=2,3,4,5$.

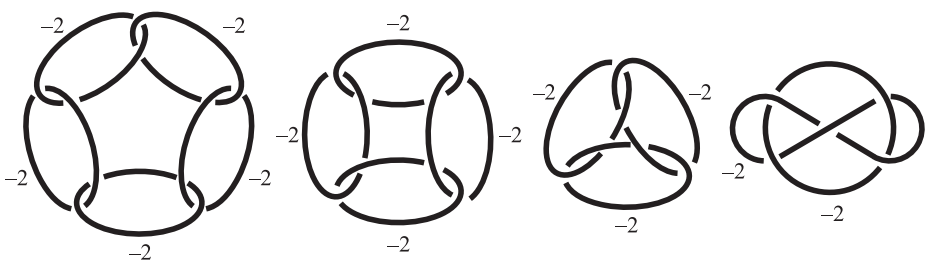

Figure 8: Four exceptional fillings that cannot be obtained by filling $F$.

with its mirror image induces on the fillings on $M_{5}$ the partial map

$$
\left(-1,-2,-2,-2, \alpha_{5}\right) \longmapsto\left(-1,-2,-2,-2,-\alpha_{5}-6\right)
$$

as one can check with SnapPy or prove using blow-downs as in figure 6 .

We can now get back to the exceptional fillings on $M_{5}$, showing that for $i=2,3,4,5$ if all the boundary components of $M_{i}$ are filled along the slope -2 , as illustrated in figure 8 , then the resulting manifold is non-hyperbolic.

Indeed, using [24] for $i=2,3$ and the recognizer [25] for $i=4,5$ we were able to prove that

$$
M_{i}(-2, \ldots,-2)=(D,(2,1),(2,-1)) \bigcup_{\left(\begin{array}{c}
-1 \\
1-i+1
\end{array}\right)}(D,(2,1),(3,1)) .
$$



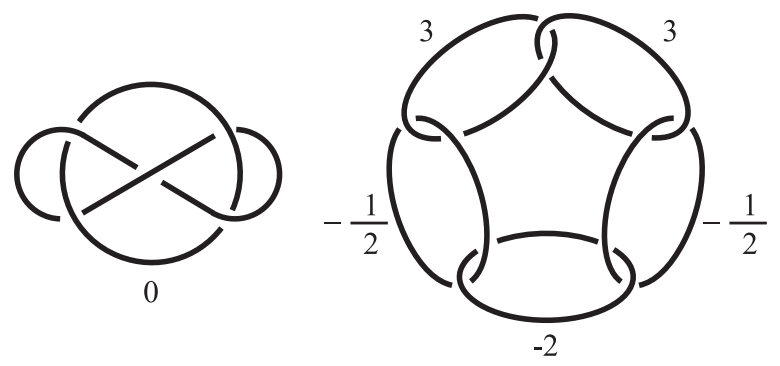

Figure 9: Two exceptional fillings of $M_{5}$ not obtained by filling $F$.

None of these manifolds can be obtained as a filling of $F=M_{5}(\infty)$, since its JSJ decomposition is that apparent in its description, and the geometric intersection number $i$ on the splitting torus of the Seifert fibrations of the two JSJ blocks is greater than 1, whereas it can only be 1 for a graph-manifold filling of $F$. Recalling that $M_{i-1}$ was recognized to be $M_{i}(-1)$ using the blow-down of figure 5 it is now easy to see that the exceptional fillings just described imply that the following elements of $\Phi^{5}$ are exceptional for $M_{5}$ :

$$
\begin{array}{ll}
(-1,-2,-2,-3,-5) & (-1,-2,-3,-2,-4) \\
(-1,-3,-2,-2,-3) & (-2,-2,-2,-2,-2)
\end{array}
$$

We conclude with two more exceptional fillings, as described in figure 9, one on $M_{2}$ and one on $M_{5}$. Using [24] and the Recognizer, the resulting manifolds were identified to be respectively

$$
M_{2}(0)=\left(P \times S^{1}\right) /_{\left(\begin{array}{ll}
0 & 1 \\
1 & 0
\end{array}\right)}, \quad M_{5}\left(-2,-\frac{1}{2}, 3,3,-\frac{1}{2}\right)=(A,(2,-1)) /_{\left(\begin{array}{ll}
1 & 2 \\
1 & 1
\end{array}\right)} .
$$

Neither of them can be obtained as a filling of $F$, and the latter cannot be obtained as a filling of the former, because the geometric intersection numbers of the Seifert fibers on the non-separating JSJ tori are different. Using the blow-down of figure 5 to identify $M_{2}(0)$ as a filling of $M_{5}$, we get the following further exceptional fillings on $M_{5}$ :

$$
(-1,-2,-2,-1) \quad\left(-2,-\frac{1}{2}, 3,3,-\frac{1}{2}\right)
$$


Exceptional Dehn surgery on the minimally twisted five-chain link 705

\subsection{The main theorem}

Quite surprisingly, the seven exceptional fillings on $M_{5}$ just described turn out to be sufficient to describe all other ones up to the symmetries of $M_{1}$, $M_{4}$ and $M_{5}$ :

Theorem 1.2. Every exceptional filling on $M_{5}$ is equivalent up to a composition of the maps (1.1) to (1.5) to a filling containing one of

$$
\begin{aligned}
& \infty \quad(-1,-2,-2,-1) \quad\left(-2,-\frac{1}{2}, 3,3,-\frac{1}{2}\right) \quad(-1,-2,-2,-3,-5) \\
& (-1,-2,-3,-2,-4) \quad(-1,-3,-2,-2,-3) \quad(-2,-2,-2,-2,-2) \text {. }
\end{aligned}
$$

Moreover, no two of these seven fillings are related to each other by any composition of the maps (1.1) to (1.5).

The exact identification given above of the manifolds obtained by filling $M_{5}$ along the 7 tuples of slopes listed in Theorem 1.2 now implies, together with the discussion in Section 1.3, that a precise description can be provided of all the manifolds arising as exceptional fillings of $M_{5}$. We will now spell this out in the closed case, i.e., for manifolds obtained by filling all 5 cusps of $M_{5}$, but the analogous statement for the case where fewer cusps are filled could be easily given:

Corollary 1.3. Every closed filling of $M_{5}$ is hyperbolic, except those listed below and those obtained from them via compositions of the maps (1.1) to $(1.5)$

$$
\begin{aligned}
M_{5}\left(\infty, \frac{a}{b}, \frac{c}{d}, \frac{e}{f}, \frac{g}{h}\right) & =(D,(a,-b),(d, c)) \bigcup_{\left(\begin{array}{ll}
0 & 1 \\
1 & 0
\end{array}\right)}(D,(f, e),(g,-h)), \\
M_{5}\left(-1,-2,-2,-1, \frac{a}{b}\right) & =(A,(b,-a-b)) /\left(\begin{array}{ll}
0 & 1 \\
1 & 0
\end{array}\right) \\
M_{5}(-1,-2,-2,-3,-5) & =(D,(2,1),(2,-1)) \bigcup_{\left(\begin{array}{cc}
-1 & 2 \\
1 & -1
\end{array}\right)}(D,(2,1),(3,1)), \\
M_{5}(-1,-2,-3,-2,-4) & =(D,(2,1),(2,-1)) \bigcup_{\left(\begin{array}{cc}
-1 & 3 \\
1 & -2
\end{array}\right)}(D,(2,1),(3,1)),
\end{aligned}
$$




$$
\begin{aligned}
& M_{5}(-1,-3,-2,-2,-3)=(D,(2,1),(2,-1)) \bigcup_{\left(\begin{array}{cc}
-1 & 4 \\
1 & -3
\end{array}\right)}(D,(2,1),(3,1)), \\
& M_{5}(-2,-2,-2,-2,-2)=(D,(2,1),(2,-1)) \bigcup_{\left(\begin{array}{cc}
-1 & 5 \\
1 & -4
\end{array}\right)}(D,(2,1),(3,1)), \\
& M_{5}\left(-2,-\frac{1}{2}, 3,3,-\frac{1}{2}\right)=(A,(2,-1)) /_{\left(\begin{array}{ll}
1 & 2 \\
1 & 1
\end{array}\right)} .
\end{aligned}
$$

Proof. We only need to check the correct expression for the filled manifolds on first two lines, depending on the filling parameters. The expression on the first line is easily derived from figure 4 , and that on the second line is taken from [24].

\section{The code}

We have written a python code, named

$$
\text { find_exceptional_fillings.py }
$$

available from [23] and based on the SnapPy libraries written by Culler et al. [9]. The code takes as an input a manifold $N$ and gives as an output a list of candidate isolated exceptional fillings of $N$. It uses the hikmot python library [15] and contains a certain ad hoc rewriting of some SnapPea routines.

We explain in this section how and why the code works; the reader who is interested only in its implementation may skip this section and read the next one.

\subsection{The algorithm}

The code follows a standard iterative algorithm, already used in [24], that we briefly recall here.

For every cusp $T$ of $N$, the code uses SnapPy to determine the cusp shape $x+\mathrm{i} y$ of $T$, in the sense that up to dilation $T$ is the quotient of $\mathbb{C}$ under the lattice generated by 1 and by $x+\mathrm{i} y$. The code also finds the area $A$ of $T$ in a maximal horospherical cusp section, where maximality is meant in the sense that all cusps of $N$ are simultaneously expanded at equal volume until they first cease to be embedded and disjoint. The code then enumerates the finitely many slopes on $T$ having length at most 6 . Note that a slope expressed by $\frac{p}{q}$ with respect to the basis of $H_{1}(T)$ corresponding to 
the generators 1 and $x+\mathrm{i} y$ of the lattice giving $T$ as a quotient has length

$$
\ell\left(\frac{p}{q}\right)=\sqrt{\frac{A}{y}\left((p+x q)^{2}+(y q)^{2}\right)} .
$$

For every slope $s$ in this finite list, the code checks whether the filled manifold $N(s)$ is hyperbolic or not. If it is, the algorithm proceeds iteratively with $N(s)$. If it is not, it appends the slope $s$ to the list of isolated exceptional fillings on $N$. Every isolated exceptional filling on $N$ is guaranteed to be detected by this algorithm thanks to the Agol-Lackenby "6-theorem" [1, 22], according to which a filling on $N$ with all slopes all having length greater than 6 is hyperbolike, and hence hyperbolic thanks to geometrization. (Perelman's general proof of the geometrization conjecture is not strictly necessary if $N$ is a hyperbolic filling of $M_{5}$, because the symmetry $\iota$ of $M_{5}$ acts on each cusp as the elliptic involution and hence extends to every filling of $M_{5}$, therefore the orbifold theorem [5] ensures geometrization for all the fillings of $M_{5}$.)

The process of listing the slopes of length at most 6 is of course sensitive to any numerical approximation in the computation of the cusp shape $x+\mathrm{i} y$ and the area $A$. To ensure rigor, our code computes these values up to some (very) small error (see below), thus guaranteeing that all slopes of length at most 6 are picked. Occasionally some slope of length more than 6 might be included, causing a little redundant analysis but not affecting the accuracy of the proof. It is also important to note that the program only produces a list of candidate isolated exceptional fillings, that one needs to verify by hand. More precisely, the code produces two lists of fillings of $N$, and each list requires some kind of a posteriori confirmation. To describe these lists we need to briefly recall how SnapPy constructs hyperbolic structures on 3-manifolds.

\subsection{Thurston's hyperbolicity equations}

We describe here the classical general strategy used to construct hyperbolic structures on 3-manifolds. Given a cusped manifold $N$ and some filling $s$ on it, Thurston's [29] method to construct a hyperbolic structure on $N(s)$ consists in taking one complex variable $z_{i}$ for each tetrahedron in an ideal triangulation of $N$, and trying to solve certain holomorphic equations in these variables. The number of equations can be reduced to be equal to the number of variables, and a hyperbolic structure on $N(s)$ is guaranteed in presence of a geometric solution, namely one with $\Im\left(z_{i}\right)>0$ for all $i$. If it exists, a geometric solution is unique. A solution with some zero or negative $\Im\left(z_{i}\right)$ corresponds (respectively) to some tetrahedra becoming flat or 
negatively oriented, and the manifold $N(s)$ is not guaranteed to be hyperbolic if such a solution is found. Experimentally, if there are only a few tetrahedra having $\Im\left(z_{i}\right) \leqslant 0$ (for instance, if there is only one), then often $N(s)$ has a hyperbolic structure anyway. Sometimes a geometric solution for the same manifold can be found by randomly modifying the triangulation. Sometimes hyperbolicity can be established by passing to some finite cover, because if some cover of a manifold is hyperbolic then the manifold also is. We have written [23] a short code named search_geometric_solutions.py that tries to guarantee hyperbolicity using these techniques if a geometric solution is not found in the first place.

\subsection{Interval arithmetic}

SnapPy uses Newton's algorithm to find an approximate numerical solution of Thurston's hyperbolicity equations. This algorithm is very efficient, but a numerical solution does not rigorously guarantee the existence of a nearby exact solution. This annoying problem was recently solved in [15]: the authors wrote a python library called hikmot, freely available from [16], that contains the function verify_hyperbolicity(). The function takes a SnapPy triangulation as an input and tries to provide a rigorous geometric solution of Thurston's equations using the Krawczyk test. If it succeeds, the function returns a geometric solution expressed via interval arithmetic: in interval arithmetic every real number is replaced by a small interval $[a, b]$ containing it, whose width $|a-b|$ is the unavoidable error. Various functions and operations easily extend from real numbers to intervals, for instance $[a, b]+[c, d]=[a+c, b+d]$, so that error propagation is automatically kept under control. The function verify_hyperbolicity() is of course not guaranteed a priori to find a geometric solution, but when it does it provides as an output some variables $z_{i}$ (actually, small rectangles in the upper halfplane) that are guaranteed to contain a geometric solution - in particular, if verify_hyperbolicity() returns a solution then the existence of a hyperbolic structure is rigorously guaranteed.

As explained above, to make the proof rigorous it is vital to list all the slopes having length at most 6 in a system of embedded and disjoint horospherical cusp sections. Including a few longer slopes is not a problem, but none of length at most 6 must be missed. The length of a slope depends on the computation of the cusp shape $x+\mathrm{i} y$ and area $A$, that SnapPy's original routines determine numerically without keeping track of error propagation. We were then forced to rewrite these routines in python, using interval arithmetic: the new routines produce intervals $A, x$ and $y$ that are guaranteed to 
contain the true values of $A, x$ and $y$. The length $\ell\left(\frac{p}{q}\right)$ of the slope $\frac{p}{q}$ is then also an interval $\left[\ell_{1}\left(\frac{p}{q}\right), \ell_{2}\left(\frac{p}{q}\right)\right]$, found using the interval-arithmetic analog of (2.1). Selecting all the slopes $\frac{p}{q}$ such that $\ell_{1}\left(\frac{p}{q}\right) \leqslant 6$ we then obtained a finite list rigorously guaranteed to contain all possibly exceptional slopes.

\subsection{Cusp area and shape}

Our routines that calculate $A, x$ and $y$ differ at some points from those used by SnapPea and we thus describe them. The first step is the construction of some (probably non-maximal) horospherical section of each cusp. Recall that the hyperbolic manifold is described by an ideal triangulation and by a geometric solution $\left(z_{i}\right)$ of Thurston's equations. Every $z_{i}$ is actually a small rectangle, but in what follows we will manipulate it as if it were a complex number, since all the operations we will use have their counterpart in interval arithmetic.

The ideal triangulation induces a triangulation of each toric boundary component. On each complete (i.e., unfilled) boundary component the code picks an arbitrary oriented edge and assigns to it the complex length 1 . The code then uses the $z_{i}$ 's to extend the complex length assignment to all the oriented edges of the torus. By construction, the complex lengths of one edge with opposite orientations are opposite to each other, and the sums of the complex edge lengths along the boundary of each oriented triangle is 0 . These complex lengths fully describe the Euclidean structure of the torus up to similarity. In addition, the real length of an edge is just the modulus of its complex length, therefore Heron's formula can be used to compute the area of each triangle and hence of the full Euclidean torus. We know that the complex lengths describe an embedded horospherical cusp section provided that the area is small enough, and for theoretical reasons [9] the universal upper bound $\frac{3}{8} \sqrt{3}$ on the area of each cusp is sufficient to ensure that all the cusp sections are embedded and disjoint. To get the desired horospherical section the code then rescales the Euclidean structure on each torus (which amounts to multiplying the complex length of every edge on the torus by a certain positive constant) until the area is $\frac{3}{8} \sqrt{3}$.

Having found the complex lengths giving the Euclidean structure on the torus, the code can now compute the cusp shape $x+\mathrm{i} y$ with respect to the fixed meridian-longitude homology basis. To do this, following SnapPea, the code describes two curves representing the meridian and the longitude as normal curves with respect to the triangulation of the toric cusp, and it computes the corresponding complex translation lengths by developing the 
triangulation along the curve. The complex shape $x+\mathrm{i} y$ is then the ratio of the translation lengths of the longitude and of the meridian.

We now turn to the computation of the area $A$ of a maximal horospherical cusp section. As we will explain soon, this is best carried out when the triangulation of the manifold is not an arbitrary one, but the canonical Epstein-Penner [11] Euclidean decomposition, or a subdivision of it, that our code then asks SnapPy to find. The triangulation that SnapPy gives as an answer is not strictly guaranteed to be the canonical one because SnapPy's computations are not rigorous, but we can take this into account. In very sporadic cases SnapPy is unable to canonize the triangulation, and in these situations we just set $A=\frac{3}{8} \sqrt{3}$, which is always fine because the existence of embedded disjoint cusp sections each having this area is guaranteed on any hyperbolic manifold. In all other cases, the code works with a triangulation which is very likely to be the canonical one, and, as detailed above, it starts by constructing a section of area $\frac{3}{8} \sqrt{3}$ at each cusp. These cusp sections lift to infinitely many horoballs in $\mathbb{H}^{3}$, and, if the triangulation is canonical, the minimal distance between two distinct such horoballs is realized along some edge of the ideal triangulation. For each edge $e$ of the triangulation the code then computes the distance $d(e)$ between the horoballs centered at the ends of $e$, to do which it picks one ideal tetrahedron of which $e$ is an edge, and it employs a nice formula to be found in the SnapPea kernel [9]. Supposing the tetrahedron has vertices $0,1,2,3$ and denoting by $e(p, q)$ the edge with ends $p$ and $q$, and by $w(r ; p, q)$ the boundary edge on the link of $r$ with ends on $e(r, p)$ and $e(r, q)$, the formula reads

$$
d(e(p, q))=-\frac{1}{2} \log (L(w(p ; r, q)) L(w(p ; s, q)) L(w(q ; r, p)) L(w(q ; s, p))),
$$

where $\{p, q, r, s\}=\{0,1,2,3\}$ and $L$ denotes the real length of a boundary edge with respect to the Euclidean structure already found. Taking the minimum $d$ of $d(e)$ over all the edges $e$ of the triangulation, one theoretically knows that by rescaling all the complex edge lengths by a factor $e^{\frac{d}{2}}$ the new minimal $d$ is then 0 , and, if the triangulation is indeed canonical, this gives a maximal horospherical cusp section with all cusps of area $A=e^{d} \times \frac{3}{8} \sqrt{3}$.

However, we are not $100 \%$ sure that the triangulation is canonical. To deal with this issue we use the fact that, for any triangulation, to guarantee that embedded and disjoint horospherical cusp sections have been found, besides checking the condition $d>0$, one must make sure that for every ideal tetrahedron $T$ and at every vertex $v$ of $T$ the three complex lengths of the boundary edges of the link of $v$ determine a triangle entirely contained in $T$. To do so, we represent $T$ in the upper half-space model of $\mathbb{H}^{3}$ as in 

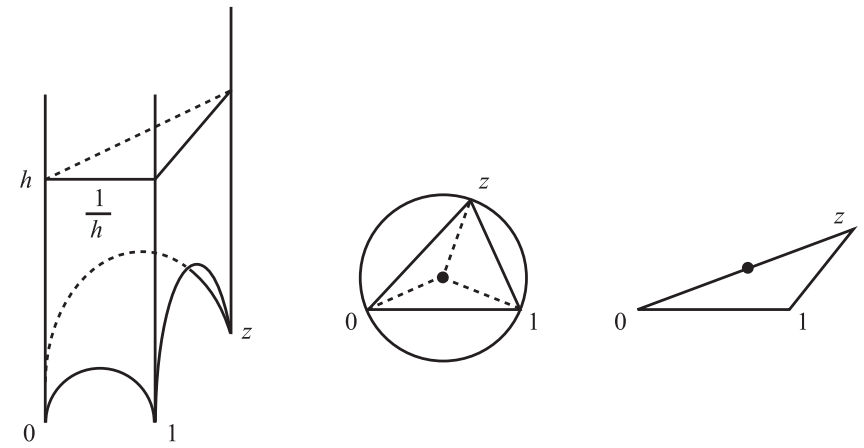

Figure 10: The horospherical section is a triangle if its height $h$ is greater than some $k$. If all the inner angles of the triangle with vertices $0,1, z$ are acute, then $k$ is the radius of the circumcircle of the triangle, otherwise $k$ is half the length of the longest edge of the triangle.

figure 10, with $v$ at $\infty$. The Euclidean height $h$ of the cusp section at $\infty$ is then easily seen to be the inverse of the length of the boundary edge whose vertices lie above 0 and 1 , and the horizontal plane at height $h$ intersects $T$ in a triangle if and only if $h$ is greater than some number $k$ that we now explain how to determine. Recall that $T$ is the intersection of four halfspaces, three bounded by vertical planes and one bounded by the hyperbolic plane whose circle at infinity $C$ contains 0,1 and $z$. If the center of $C$ lies in the triangle of vertices 0,1 and $z$ then $k$ equals the radius of $C$, otherwise $k$ equals $\max \left\{\frac{1}{2}, \frac{|z|}{2}, \frac{|z-1|}{2}\right\}$. Our code makes sure that embedded and disjoint horospherical cusp sections have been found by checking that $h>k$ for every choice of $T$ and $v$, taking errors into account. The code is actually designed to return $A=\frac{3}{8} \sqrt{3}$ as above in case some test $h>k$ fails, but as a matter of fact this never happened during our computations (which is not surprising, since the triangulation $T$ is extremely likely to be the canonical one or a subdivision of it).

\section{Proof of the main theorem}

In this section we describe our proof of Theorem 1.2, that, as anticipated above, is computer-assisted but rigorous. Our main tool is the python code find_exceptional_fillings.py described in the previous section and publicly available from [23]. 


\subsection{The output}

The code find_exceptional_fillings.py takes a cusped hyperbolic manifold $N$ as an input and produces two lists as an output:

(I) A list of candidate exceptional fillings of $N$, for which SnapPy was unable to find any kind of solution.

(II) A list of candidate hyperbolic fillings of $N$, for which SnapPy found some numerical non-geometric solution (with flat or negatively oriented tetrahedra).

Typically, list (II) consists of closed manifolds only. If this is the case, the classification of the exceptional fillings of $N$ then becomes complete and rigorous provided we can a posteriori confirm the following:

(i) all the manifolds in list (I) are non-hyperbolic and

(ii) all the manifolds in list (II) are hyperbolic.

On the contrary, when list (II) contains some manifold $Y$ with boundary tori, (ii) is necessary but not sufficient, because we should separately test for hyperbolicity the fillings of $Y$. However, for all the $N$ 's we had to deal with in the proof of Theorem 1.2, list (II) indeed contained closed manifolds only.

\subsection{Organizing the search}

Despite our main objective being that to establish Theorem 1.2, we have not run our code directly with $M_{5}$ as an input, because it turned out that considerable computer time could be saved by dealing first with the manifolds $M_{2}, M_{3}$ (thereby confirming the results of [24]) and $M_{4}$, and only then with $M_{5}$. In the next pages, along with the description of the data obtained step after step, we will give an explanation of the exact strategy we have used to employ the data obtained at one step to make the next step simpler.

\subsection{The Whitehead link exterior}

Running the code for $M_{2}$ (the exterior of the Whitehead link), we get for this manifold the lists (I) and (II) described above. With a slope $\frac{p}{q} \neq \emptyset$ written as $(p, q)$, the empty slope written as $(0,0)$, and pairs of slopes (used to fill 
Exceptional Dehn surgery on the minimally twisted five-chain link 713

the two boundary components of $M_{2}$ ) written between square brackets, the output is as follows:

Candidate exceptional fillings:

With 1 fillings:

$[[(0,0),(0,1)],[(0,0),(1,0)],[(0,0),(1,1)],[(0,0),(2,1)]$,

$[(0,0),(3,1)],[(0,0),(4,1)],[(0,1),(0,0)],[(1,0),(0,0)]$,

$[(1,1),(0,0)],[(2,1),(0,0)],[(3,1),(0,0)],[(4,1),(0,0)]]$

Total: 12

With 2 fillings:

$[[(-4,1),(-1,1)],[(-3,1),(-1,1)],[(-2,1),(-2,1)]$,

$[(-2,1),(-1,1)],[(-1,1),(-4,1)],[(-1,1),(-3,1)]$,

$[(-1,1),(-2,1)],[(-1,1),(-1,1)],[(3,2),(5,1)]$,

$[(4,3),(5,1)],[(5,1),(3,2)],[(5,1),(4,3)]$,

$[(5,2),(7,2)],[(7,2),(5,2)]]$

Total: 14

Candidate hyperbolic fillings:

With 1 fillings:

[]

Total: 0

With 2 fillings:

$[[(-3,1),(-2,1)],[(-2,1),(-3,1)],[(3,2),(6,1)]$, $[(5,2),(6,1)],[(6,1),(3,2)],[(6,1),(5,2)]]$

Total: 6

Getting back to our tasks, we first simplify (i) - the confirmation that the candidate exceptional fillings are indeed non-hyperbolic - by noting that there is a symmetry of $M_{2}$ that switches the two boundary components, with trivial action on slopes given by

$$
\left(\alpha_{1}, \alpha_{2}\right) \mapsto\left(\alpha_{2}, \alpha_{1}\right) .
$$

Using (3.1) we see that to achieve (i) we must only show that the fillings

$$
\begin{array}{llllll}
0 & 1 & 2 & 3 & 4 & \infty
\end{array}
$$

on one component, and

$$
\begin{aligned}
& (-2,-2) \quad(-1,-4) \\
& (-1,-3) \quad(-1,-2) \\
& (-1,-1) \quad\left(\frac{4}{3}, 5\right) \\
& \left(\frac{3}{2}, 5\right) \quad\left(\frac{5}{2}, \frac{7}{2}\right)
\end{aligned}
$$


on both components are truly exceptional. Exceptionality of these fillings was already proved in [24], and we can actually make the list shorter using the additional symmetries

$$
\begin{aligned}
\left(-1, \alpha_{2}\right) & \mapsto\left(-1,-\alpha_{2}\right), \\
\left(5, \alpha_{2}\right) & \mapsto\left(5, \frac{\alpha_{2}}{\alpha_{2}-1}\right)
\end{aligned}
$$

that follow from the amphichirality of $M_{2}(-1)$ and $M_{2}(5)$ - the figureeight knot exterior and its sibling — and are detected by SnapPy. Under the action of these maps the set of exceptional fillings on $M_{2}$ reduces to

$$
\begin{array}{lllllll}
0 & 1 & 2 & 3 & 4 & \infty & (-2,-2)
\end{array}\left(\frac{5}{2}, \frac{7}{2}\right)
$$

as summarized in table 2. Getting to task (ii) - the confirmation that the candidate hyperbolic fillings are indeed hyperbolic — up to (3.1) we must prove hyperbolicity only of

$$
(-2,-3) \quad\left(\frac{3}{2}, 6\right) \quad\left(\frac{5}{2}, 6\right)
$$

which we can do using search_geometric_solution. In all three cases the code finds a geometric solution on some finite cover of degree at most 10 . Note that $M_{2}\left(\frac{3}{2}, 6\right)$ is the closed hyperbolic manifold Vol3 analyzed in [20], for which no geometric solution is known. We have thus rigorously proved the following:

Theorem 3.1. Every exceptional Dehn filling on $M_{2}$ is equivalent up to a composition of the maps (3.1) to (3.3) to a filling containing one of

$$
\begin{array}{lllllll}
0 & 1 & 2 & 3 & 4 & \infty & (-2,-2)
\end{array}\left(\frac{5}{2}, \frac{7}{2}\right) .
$$

Moreover, no two of these eight fillings are related to each other by any composition of the maps (3.1) to (3.3).

Remark 3.2. The last assertion of the previous theorem is readily proved by direct inspection, and we will refrain from stating the analogous ones in the subsequent Theorems 3.3 and 3.5. 
Exceptional Dehn surgery on the minimally twisted five-chain link 715

\subsection{The magic manifold}

After the Whitehead link exterior, the next item in the sequence $\left(M_{i}\right)_{i=1}^{5}$ introduced in Section 1.4 is the magic manifold $M_{3}$. The exceptional fillings on $M_{3}$ were already classified in [24], and using our code we can rigorously confirm this classification along the lines explained above. Since $M_{2}$ is obtained by a (-1)-filling on any component of $M_{3}$ and the exceptional fillings on $M_{2}$ are those in Theorem 3.1, we can exclude from our search any filling on $M_{3}$ containing a -1 . In the code find_exceptional_fillings.py there is a list named exclude that by default is empty and that for $M_{3}$ we set as

exclude $=[[(-1,1),(0,0),(0,0)],[(0,0),(-1,1),(0,0)]$, $[(0,0),(0,0),(-1,1)]]$

then getting the following as an output:

Candidate exceptional fillings:

With 1 fillings:

$[[(0,0),(0,0),(0,1)],[(0,0),(0,0),(1,0)],[(0,0),(0,0),(1,1)]$, $[(0,0),(0,0),(2,1)],[(0,0),(0,0),(3,1)],[(0,0),(0,1),(0,0)]$, $[(0,0),(1,0),(0,0)],[(0,0),(1,1),(0,0)],[(0,0),(2,1),(0,0)]$, $[(0,0),(3,1),(0,0)],[(0,1),(0,0),(0,0)],[(1,0),(0,0),(0,0)]$, $[(1,1),(0,0),(0,0)],[(2,1),(0,0),(0,0)],[(3,1),(0,0),(0,0)]]$

Total: 15

With 2 fillings:

$[[(0,0),(1,2),(4,1)],[(0,0),(3,2),(5,2)],[(0,0),(4,1),(1,2)]$, $[(0,0),(5,2),(3,2)],[(1,2),(0,0),(4,1)],[(1,2),(4,1),(0,0)]$, $[(3,2),(0,0),(5,2)],[(3,2),(5,2),(0,0)],[(4,1),(0,0),(1,2)]$, $[(4,1),(1,2),(0,0)],[(5,2),(0,0),(3,2)],[(5,2),(3,2),(0,0)]]$

Total: 12

With 3 fillings:

$[[(-2,1),(-2,1),(-2,1)],[(1,2),(5,1),(5,1)]$, $[(2,3),(4,1),(4,1)],[(3,2),(3,2),(4,1)],[(3,2),(3,2),(8,3)]$, $[(3,2),(4,1),(3,2)],[(3,2),(7,3),(7,3)],[(3,2),(8,3),(3,2)]$, $[(4,1),(2,3),(4,1)],[(4,1),(3,2),(3,2)],[(4,1),(4,1),(2,3)]$, $[(4,3),(5,2),(5,2)],[(5,1),(1,2),(5,1)],[(5,1),(5,1),(1,2)]$, $[(5,2),(4,3),(5,2)],[(5,2),(5,2),(4,3)],[(5,2),(5,3),(5,3)]$, $[(5,3),(5,2),(5,3)],[(5,3),(5,3),(5,2)],[(7,3),(3,2),(7,3)]$, $[(7,3),(7,3),(3,2)],[(8,3),(3,2),(3,2)]]$

Total: 22 
Candidate hyperbolic fillings:

With 1 fillings:

[]

Total: 0

With 2 fillings:

[]

Total: 0

With 3 fillings:

$[[(-3,1),(-2,1),(-2,1)],[(-3,1),(-2,1),(4,1)]$,

$[(-2,1),(-3,1),(-2,1)],[(-2,1),(-2,1),(-3,1)]$,

$[(-2,1),(1,2),(3,2)],[(2,3),(4,1),(-2,1)]$,

$[(4,3),(3,2),(4,1)],[(4,3),(4,1),(3,2)]$,

$[(5,1),(3,2),(3,2)],[(5,3),(3,2),(4,1)]$,

$[(5,3),(4,1),(3,2)],[(7,3),(3,2),(3,2)]$,

$[(7,3),(3,2),(5,3)]]$

Total: 13

To proceed we note that any element of the permutation group $\mathfrak{S}_{3}$ on the cusps of $M_{3}$ is realized by an isometry of $M_{3}$, with action on the slopes generated by two transpositions:

$$
\begin{aligned}
&\left(\alpha_{1}, \alpha_{2}, \alpha_{3}\right) \mapsto\left(\alpha_{3}, \alpha_{2}, \alpha_{1}\right), \\
&\left(\alpha_{1}, \alpha_{2}, \alpha_{3}\right) \mapsto\left(\alpha_{2}, \alpha_{1}, \alpha_{3}\right) .
\end{aligned}
$$

In addition, as already stated in [24], a few fillings of $M_{3}$ have additional symmetries, inducing the following partial maps on the slopes:

$$
\begin{aligned}
\left(\frac{1}{2}, \alpha_{2}, \alpha_{3}\right) & \mapsto\left(\frac{1}{2}, 4-\alpha_{2}, 4-\alpha_{3}\right), \\
\left(\frac{3}{2}, \alpha_{2}, \alpha_{3}\right) & \mapsto\left(\frac{3}{2}, \frac{2 \alpha_{2}-5}{\alpha_{2}-2}, \frac{2 \alpha_{3}-5}{\alpha_{3}-2}\right), \\
\left(\frac{5}{2}, \alpha_{2}, \alpha_{3}\right) & \mapsto\left(\frac{5}{2}, \frac{\alpha_{2}-3}{\alpha_{2}-2}, \frac{2 \alpha_{3}-3}{\alpha_{3}-1}\right), \\
\left(4, \alpha_{2}, \alpha_{3}\right) & \mapsto\left(4, \frac{\alpha_{2}-2}{\alpha_{2}-1}, \frac{\alpha_{3}-2}{\alpha_{3}-1}\right), \\
\left(-1,-2, \alpha_{3}\right) & \mapsto\left(-1,-2,-\alpha_{3}-2\right), \\
\left(-1,4, \alpha_{3}\right) & \mapsto\left(-1,4, \frac{1}{\alpha_{3}}\right) .
\end{aligned}
$$


Note that (3.10) and (3.11) follow from (3.2) and (3.3) because $M_{3}(-1$, $\left.\alpha_{2}, \alpha_{3}\right)=M_{2}\left(\alpha_{2}+1, \alpha_{3}+1\right)$, whereas (3.3) induces at the level of $M_{3}$ a map that is obtained (in a complicated fashion) by the other maps listed. We now embed the achievement of tasks (i)-(iii) for $M_{3}$ in the proof of the following result, that confirms the main statement of [24] — with an overall change of sign because the chain link considered there is the mirror image of the one considered here:

Theorem 3.3. Every exceptional Dehn filling on $M_{3}$ is equivalent up to a composition of the maps (3.4) to (3.11) to a filling containing one of

$$
\begin{array}{lllllllll}
0 & 1 & 2 & 3 & \infty & (-1,-1) & (-1,-3,-3) & (-2,-2,-2) & \left(\frac{2}{3}, 4,4\right) .
\end{array}
$$

Proof. All the candidate exceptional fillings on $M_{3}$ contained in the list (I) produced by our code for $M_{3}$ had already been precisely recognized and hence proved to be indeed exceptional in [24], which completes task (i) for $M_{3}$. Letting the maps (3.4) and (3.5) act we reduce the list to

$$
\begin{array}{ccccc}
0 & 1 & 2 & 3 & \infty \\
\left(\frac{1}{2}, 4\right) & \left(\frac{3}{2}, \frac{5}{2}\right) & (-2,-2,-2) & \left(\frac{1}{2}, 5,5\right) & \left(\frac{2}{3}, 4,4\right) \\
\left(\frac{4}{3}, \frac{5}{2}, \frac{5}{2}\right) & \left(\frac{3}{2}, \frac{7}{3}, \frac{7}{3}\right) & \left(\frac{3}{2}, \frac{3}{2}, \frac{8}{3}\right) & \left(\frac{3}{2}, \frac{3}{2}, 4\right) & \left(\frac{5}{3}, \frac{5}{3}, \frac{5}{2}\right)
\end{array}
$$

to which we add

$$
\begin{aligned}
(-1,-1) & (-1,-3,-3)(-1,-2,-5) \quad(-1,-2,-4) \\
(-1,-2,-3) & (-1,-2,-2)\left(-1, \frac{1}{3}, 4\right)
\end{aligned}
$$

coming from the isolated exceptional fillings on $M_{2}=M_{3}(-1)$ found in Theorem 3.1, under the identification $M_{3}\left(-1, \alpha_{2}, \alpha_{3}\right)=M_{2}\left(\alpha_{2}+1, \alpha_{3}+1\right)$ given by the blow-down move of figure 5 . This is in complete agreement with [24], and letting all the maps (3.6) to (3.11) act we now get the list of nine exceptional fillings in the statement.

Turning to task (ii), the code search_geometric_solutions.py succeeds in finding a geometric solution for each candidate hyperbolic filling of list (II) for $M_{3}$. 


\subsection{The 4-chain link exterior}

Our next aim is to classify the exceptional fillings on the exterior $M_{4}$ of the chain link with four components shown in figure 2. The symmetry group of this link is isomorphic to $D_{4} \times \mathbb{Z} / 2$, with the factor $\mathbb{Z} / 2$ generated by an involution similar to the $\iota$ described in figure 3 , with trivial action on fillings, and two generators of the dihedral group $D_{4}$ act on fillings as follows:

$$
\begin{aligned}
&\left(\alpha_{1}, \alpha_{2}, \alpha_{3}, \alpha_{4}\right) \longmapsto\left(\alpha_{4}, \alpha_{1}, \alpha_{2}, \alpha_{3}\right), \\
&\left(\alpha_{1}, \alpha_{2}, \alpha_{3}, \alpha_{4}\right) \longmapsto\left(\alpha_{4}, \alpha_{3}, \alpha_{2}, \alpha_{1}\right) .
\end{aligned}
$$

In addition to these symmetries coming from the link, the symmetry group of $M_{4}$ contains a $\mathbb{Z} / 2 \times \mathbb{Z} / 2$ subgroup leaving each cusp invariant, with two generators acting as follows on slopes:

$$
\begin{aligned}
& \left(\alpha_{1}, \alpha_{2}, \alpha_{3}, \alpha_{4}\right) \longmapsto\left(\frac{\alpha_{1}-2}{\alpha_{1}-1}, \frac{\alpha_{2}-2}{\alpha_{2}-1}, \frac{\alpha_{3}-2}{\alpha_{3}-1}, \frac{\alpha_{4}-2}{\alpha_{4}-1}\right), \\
& \left(\alpha_{1}, \alpha_{2}, \alpha_{3}, \alpha_{4}\right) \longmapsto\left(2-\alpha_{1}, \frac{\alpha_{2}}{\alpha_{2}-1}, 2-\alpha_{3}, \frac{\alpha_{4}}{\alpha_{4}-1}\right) .
\end{aligned}
$$

Before running our code on $M_{4}$ we can now exclude the filling - 1 , that gives $M_{3}$, and all the fillings obtained from -1 under compositions of the maps (3.12) to (3.15), which give $-1, \frac{3}{2}, 3, \frac{1}{2}$ on each cusp, for a total of 16 slopes. In find_exceptional_fillings.py we then modify the list exclude to

$$
\begin{aligned}
& \text { exclude }=[ \\
& {[(-1,1),(0,0),(0,0),(0,0)],[(3,2),(0,0),(0,0),(0,0)],} \\
& {[(3,1),(0,0),(0,0),(0,0)],[(1,2),(0,0),(0,0),(0,0)],} \\
& {[(0,0),(0,0),(0,0),(-1,1)],[(0,0),(0,0),(0,0),(3,2)],} \\
& {[(0,0),(0,0),(0,0),(3,1)],[(0,0),(0,0),(0,0),(1,2)],} \\
& {[(0,0),(-1,1),(0,0),(0,0)],[(0,0),(3,2),(0,0),(0,0)],} \\
& {[(0,0),(3,1),(0,0),(0,0)],[(0,0),(1,2),(0,0),(0,0)],} \\
& {[(0,0),(0,0),(-1,1),(0,0)],[(0,0),(0,0),(3,2),(0,0)],} \\
& [(0,0),(0,0),(3,1),(0,0)],[(0,0),(0,0),(1,2),(0,0)]]
\end{aligned}
$$

getting the following output:

Candidate exceptional fillings:

With 1 fillings:

$[[(0,0),(0,0),(0,0),(0,1)],[(0,0),(0,0),(0,0),(1,0)]$,

$[(0,0),(0,0),(0,0),(1,1)],[(0,0),(0,0),(0,0),(2,1)]$, 
Exceptional Dehn surgery on the minimally twisted five-chain link 719

$[(0,0),(0,0),(0,1),(0,0)],[(0,0),(0,0),(1,0),(0,0)]$,
$[(0,0),(0,0),(1,1),(0,0)],[(0,0),(0,0),(2,1),(0,0)]$,
$[(0,0),(0,1),(0,0),(0,0)],[(0,0),(1,0),(0,0),(0,0)]$,
$[(0,0),(1,1),(0,0),(0,0)],[(0,0),(2,1),(0,0),(0,0)]$,
$[(0,1),(0,0),(0,0),(0,0)],[(1,0),(0,0),(0,0),(0,0)]$,
$[(1,1),(0,0),(0,0),(0,0)],[(2,1),(0,0),(0,0),(0,0)]]$

Total: 16

With 2 fillings:

[]

Total: 0

With 3 fillings:

[]

Total: 0

With 4 fillings:

$[[(-2,1),(-2,1),(-2,1),(-2,1)],[(2,3),(4,1),(3,4),(4,1)]$, $[(4,1),(2,3),(4,1),(2,3)],[(4,3),(4,3),(4,3),(4,3)]]$

Total: 4

Candidate hyperbolic fillings:

With 1 fillings:

[]

Total: 0

With 2 fillings:

[]

Total: 0

With 3 fillings:

[]

Total: 0

With 4 fillings:

$[[(2,3),(-2,1),(2,3),(4,1)],[(2,3),(4,1),(2,3),(5,1)]$,

$[(2,3),(4,1),(3,4),(4,1)],[(3,4),(4,1),(2,3),(4,1)]$,

$[(4,1),(-2,1),(-2,1),(-2,1)],[(4,1),(2,3),(-2,1),(2,3)]$,

$[(4,1),(2,3),(4,1),(3,4)],[(4,1),(3,4),(4,1),(2,3)]$,

$[(4,1),(4,3),(4,1),(2,3)],[(4,3),(4,3),(4,3),(5,4)]$,

$[(4,3),(4,3),(5,4),(4,3)],[(5,4),(4,3),(4,3),(4,3)]]$

Total: 12

The list (I) of candidate exceptional fillings is then very short, and up to the action of (3.12) to (3.15) it actually reduces to

$$
0 \infty \quad(-2,-2,-2,-2)
$$


which are indeed all exceptional: we have $M_{4}(\infty)=M_{5}(-1, \infty)$ and $M_{4}(0)=$ $M_{5}(-1, \emptyset, 0)$, and we know that $\infty$ and 0 are exceptional for $M_{5}$; moreover the exceptionality of $(-2,-2,-2,-2)$ was proved using the Recognizer [25], as discussed in Section 1.4. To state our classification result for the exceptional fillings of $M_{4}$ we now note that under the identification

$$
M_{4}\left(-1, \alpha_{2}, \alpha_{3}, \alpha_{4}\right)=M_{3}\left(\alpha_{2}+1, \alpha_{3}, \alpha_{4}+1\right)
$$

the maps (3.5) and (3.10) induce for $M_{4}$, respectively

$$
\begin{aligned}
\left(-1, \alpha_{2}, \alpha_{3}, \alpha_{4}\right) & \mapsto\left(-1, \alpha_{3}-1, \alpha_{2}+1, \alpha_{4}\right), \\
\left(-1,-2,-2, \alpha_{4}\right) & \mapsto\left(-1,-2,-2,-\alpha_{4}-4\right) .
\end{aligned}
$$

Proposition 3.4. Under the correspondence

$$
\left(\alpha_{1}, \alpha_{2}, \alpha_{3}\right) \leadsto\left(-1, \alpha_{1}-1, \alpha_{2}, \alpha_{3}-1\right)
$$

the action of the maps (3.4) to (3.11) on triples is generated by the action of (3.12) to (3.17) on 4-tuples.

Proof. It is very easy to see that (3.4) is induced by a combination of (3.12) and (3.13), while (3.5) is (3.16), therefore the action of (3.12) to (3.17) generates the whole isometry group (3.4) and (3.5) of $M_{3}$. To show that also (3.6) to (3.11) get generated, we first note that as a composition of (3.14) and (3.15) we get

$$
\left(\alpha_{1}, \alpha_{2}, \alpha_{3}, \alpha_{4}\right) \longmapsto\left(\frac{\alpha_{1}}{\alpha_{1}-1}, 2-\alpha_{2}, \frac{\alpha_{3}}{\alpha_{3}-1}, 2-\alpha_{4}\right) .
$$

We then start with (3.6). Up to (3.4) and (3.5), that we already know to come from (3.12) to (3.17), we can rewrite it as

$$
\left(\alpha_{2}, \frac{1}{2}, \alpha_{3}\right) \mapsto\left(4-\alpha_{3}, \frac{1}{2}, 4-\alpha_{2}\right)
$$

and then we can generate it as follows:

$$
\begin{aligned}
& \text { (3.6): }\left(\alpha_{2}, \frac{1}{2}, \alpha_{3}\right) \rightsquigarrow\left(-1, \alpha_{2}-1, \frac{1}{2}, \alpha_{3}-1\right) \\
& \underset{(3.18)}{\mapsto}\left(\frac{1}{2}, 3-\alpha_{2},-1,3-\alpha_{3}\right) \\
& \underset{(3.12)^{2}}{\mapsto}\left(-1,3-\alpha_{3}, \frac{1}{2}, 3-\alpha_{2}\right) \rightsquigarrow\left(4-\alpha_{3}, \frac{1}{2}, 4-\alpha_{2}\right) \text {. }
\end{aligned}
$$


Exceptional Dehn surgery on the minimally twisted five-chain link 721

We next similarly realize (3.7) and (3.8), again only up to (3.4) and (3.5)

$$
\begin{aligned}
& \text { (3.7) : }\left(\alpha_{2}, \frac{3}{2}, \alpha_{3}\right) \leadsto\left(-1, \alpha_{2}-1, \frac{3}{2}, \alpha_{3}-1\right) \underset{(3.14)}{\stackrel{\leftrightarrow}{(})}\left(\frac{3}{2}, \frac{\alpha_{2}-3}{\alpha_{2}-2},-1, \frac{\alpha_{3}-3}{\alpha_{3}-2}\right) \\
& \underset{(3.12)^{2}}{\mapsto}\left(-1, \frac{\alpha_{3}-3}{\alpha_{3}-2}, \frac{3}{2}, \frac{\alpha_{2}-3}{\alpha_{2}-2}\right) \leadsto\left(\frac{2 \alpha_{3}-5}{\alpha_{3}-2}, \frac{3}{2}, \frac{2 \alpha_{2}-5}{\alpha_{2}-2}\right), \\
& \text { (3.8): }\left(\frac{5}{2}, \alpha_{2}, \alpha_{3}\right) \text { m }\left(-1, \frac{3}{2}, \alpha_{2}, \alpha_{3}-1\right) \underset{(3.14)}{\stackrel{\leftrightarrow}{(}}\left(\frac{3}{2},-1, \frac{\alpha_{2}-2}{\alpha_{2}-1}, \frac{\alpha_{3}-3}{\alpha_{3}-2}\right) \\
& \underset{(3.12)^{3}}{\mapsto}\left(-1, \frac{\alpha_{2}-2}{\alpha_{2}-1}, \frac{\alpha_{3}-3}{\alpha_{3}-2}, \frac{3}{2}\right) \leadsto\left(\frac{2 \alpha_{2}-3}{\alpha_{2}-1}, \frac{\alpha_{3}-3}{\alpha_{3}-2}, \frac{5}{2}\right) .
\end{aligned}
$$

We now observe that (3.12) to (3.17) induce on triples the map

$$
\begin{array}{r}
\left(4, \alpha_{2}, \alpha_{3}\right) \leadsto\left(-1,3, \alpha_{2}, \alpha_{3}-1\right) \underset{(3.18)}{\mapsto}\left(\frac{1}{2},-1, \frac{\alpha_{2}}{\alpha_{2}-1}, 3-\alpha_{3}\right) \\
\underset{(3.12)^{3}}{\mapsto}\left(-1, \frac{\alpha_{2}}{\alpha_{2}-1}, 3-\alpha_{3}, \frac{1}{2}\right) \leadsto\left(\frac{2 \alpha_{2}-1}{\alpha_{2}-1}, 3-\alpha_{3}, \frac{3}{2}\right)
\end{array}
$$

and it is easy to see that (3.9) is obtained by conjugating (3.7) under (3.19). Moreover we can generate (3.11) as

$$
\left(-1,4, \alpha_{3}\right) \underset{(3.19)}{\mapsto}\left(\frac{3}{2}, \frac{3}{2}, 3-\alpha_{3}\right) \underset{(3.7)}{\mapsto}\left(\frac{3}{2}, 4, \frac{1-2 \alpha_{3}}{1-\alpha_{3}}\right) \underset{(3.9)}{\mapsto}\left(-1,4, \frac{1}{\alpha_{3}}\right)
$$

and the conclusion eventually follows because (3.10) is (3.17).

Theorem 3.5. Every exceptional filling on $M_{4}$ is equivalent up to a composition of the maps (3.12) to (3.17) to a filling containing one of

$$
\begin{gathered}
0 \quad(-1,-2,-1) \quad(-2,-2,-2,-2) \\
(-1,-3,-2,-3) \quad(-1,-2,-3,-4) .
\end{gathered}
$$

Proof. The code search_geometric_solutions.py shows that the 12 candidate hyperbolic manifolds in list (II) are indeed hyperbolic. We know from above that up to (3.12) to (3.15) an exceptional filling $\left(\alpha_{1}, \alpha_{2}, \alpha_{3}, \alpha_{4}\right)$ on $M_{4}$ either contains one of $0, \infty,(-2,-2,-2,-2)$ or $\alpha_{1}=-1$ and $\left(\alpha_{2}+\right.$ $\left.1, \alpha_{3}, \alpha_{4}+1\right)$ is exceptional for $M_{3}$. In the latter case by Theorem 3.3 we have that $\left(\alpha_{2}+1, \alpha_{3}, \alpha_{4}+1\right)$ contains one of

$$
\begin{array}{lllllllll}
0 & 1 & 2 & 3 & \infty & (-1,-1) & (-1,-3,-3) & (-2,-2,-2) & \left(\frac{2}{3}, 4,4\right)
\end{array}
$$

up to the maps (3.4) to (3.11). Proposition 3.4 shows that the action of these maps is generated by (3.12) to (3.17). We readily deduce that up to (3.12) 
to $(3.17)$ an exceptional $\left(-1, \alpha_{2}, \alpha_{3}, \alpha_{4}\right)$ for $M_{4}$ contains one of

$$
\begin{aligned}
& (-1,-1) \quad(-1,0) \quad(-1,1) \quad(-1,2) \quad(-1, \infty) \quad(-1,-2,-1) \\
& (-1,-2,-3,-4) \quad(-1,-3,-2,-3) \quad\left(-1,3, \frac{2}{3}, 3\right) \text {. }
\end{aligned}
$$

We can then dismiss $(-1,0),(-1,1),(-1,2)$ and $(-1, \infty)$ because we see that $0,1,2, \infty$ are exceptional on $M_{4}$ : we know that $\infty$ and 0 are, while 1 is generated by $\infty$ and 2 is generated by 0 under both (3.14) and (3.15). Using (3.16) we can also transform $(-1,-1)$ into $(-1, \emptyset, 0)$ and then dismiss it. Finally, using (3.18) we can transform $\left(-1,3, \frac{2}{3}, 3\right)$ into $\left(\frac{1}{2},-1,-2,-1\right)$ and dismiss it because it contains $(-1,-2,-1)$, and the proof is complete.

Corollary 3.6. Every filling on $M_{4}$ is hyperbolic, except those listed below and those obtained from them via compositions of the maps (3.12) to (3.17)

$$
\begin{aligned}
M_{4}\left(\infty, \frac{a}{b}, \frac{c}{d}, \frac{e}{f}\right) & =\left(S^{2},(a, b),(d,-c),(e, f)\right), \\
M_{4}\left(0, \frac{a}{b}, \frac{c}{d}, \frac{e}{f}\right) & =(D,(b, b-a),(f, f-e)) \bigcup_{\left(\begin{array}{ll}
0 & 1 \\
1 & 0
\end{array}\right)}(D,(2,-1),(c-2 d, c-d)), \\
M_{4}\left(-1,-2,-1, \frac{a}{b}\right) & =\left.(A,(b,-a))\right|_{\left(\begin{array}{ll}
0 & 1 \\
1 & 0
\end{array}\right)}, \\
M_{4}(-1,-2,-3,-4) & =(D,(2,1),(2,-1)) \bigcup_{\left(\begin{array}{cc}
-1 & 2 \\
1 & -1
\end{array}\right)}(D,(2,1),(3,1)), \\
M_{4}(-1,-3,-2,-3) & =(D,(2,1),(2,-1)) \bigcup_{\left(\begin{array}{cc}
-1 & 3 \\
1 & -2
\end{array}\right)}(D,(2,1),(3,1)), \\
M_{4}(-2,-2,-2,-2) & =(D,(2,1),(2,-1)) \bigcup_{\left(\begin{array}{cc}
-1 & 4 \\
1 & -3
\end{array}\right)}(D,(2,1),(3,1)) .
\end{aligned}
$$

Proof. We only need to check the correct expression for the filled manifold on the first three lines depending on the parameters $a, b, \ldots, f$. The equation on the first line follows by expressing $M_{4}(\infty)$ as an open chain link with 
Exceptional Dehn surgery on the minimally twisted five-chain link 723

three components. For the equation on the second line we have

$$
\begin{aligned}
M_{4}\left(0, \frac{a}{b}, \frac{c}{d}, \frac{e}{f}\right) & =M_{5}\left(0, \frac{a-b}{b},-1, \frac{c-d}{d}, \frac{e}{f}\right) \\
& =M_{5}\left(\frac{b}{a-b}, \infty, 2, \frac{c-d}{c-2 d}, \frac{f-e}{f}\right) \\
& =M_{5}\left(\infty, \frac{b}{a-b}, \frac{f-e}{f}, \frac{c-d}{c-2 d}, 2\right) \\
& =(D,(b, b-a),(f, f-e)) \bigcup_{\left(\begin{array}{ll}
0 & 1 \\
1 & 0
\end{array}\right)}(D,(2,-1),(c-2 d, c-d))
\end{aligned}
$$

using (1.3) and Corollary 1.3. Finally the equation on the third line also follows from Corollary 1.3 or [24].

\subsection{The minimally twisted 5-chain link}

We eventually prove here Theorem 1.2, concerning the isolated exceptional fillings on $M_{5}$. Recall first that $M_{5}$ decomposes into 10 regular ideal hyperbolic tetrahedra, and this decomposition is totally symmetric, so each cusp section decomposes into eight equilateral triangles. More precisely, with respect to the meridian-longitude homology basis of the cusp, its shape is given by $-\frac{1}{2}+\mathrm{i} \frac{\sqrt{3}}{2}$, and it is quite easy to see that the area of each cusp in a maximal horospherical cusp section is equal to $A=2 \sqrt{3}$, because each individual equilateral triangle has Euclidean area $\frac{\sqrt{3}}{4}$. The length of a slope $\frac{p}{q}$ is hence

$$
\ell\left(\frac{p}{q}\right)=\sqrt{4\left(\left(p-\frac{q}{2}\right)^{2}+3\left(\frac{q}{2}\right)^{2}\right)}=2 \sqrt{p^{2}+q^{2}-p q} .
$$

The slopes having length at most 6 are therefore

$$
\infty \quad-2 \quad-1 \quad-\frac{1}{2} \quad 0 \quad \frac{1}{3} \quad \frac{1}{2} \quad \frac{2}{3} \quad 1 \quad \frac{3}{2} \quad 2 \quad 3 .
$$

Recall now that the action on slopes of the symmetry group of $M_{5}$ is generated by (1.1) to (1.3); of course (1.1) and (1.2) act trivially on the set of slopes of length less than 6 just enumerated, but (1.3) allows to group them as

$$
\{\infty, 0,1\}, \quad\left\{-1, \frac{1}{2}, 2\right\}, \quad\left\{-2,-\frac{1}{2}, \frac{1}{3}, \frac{2}{3}, \frac{3}{2}, 3\right\} .
$$

Now, we already know that the slopes in the first set are exceptional and those in the second set are not, since they give $M_{4}$ as a filling. SnapPy 
tells us that $M_{5}(-2)$ is hyperbolic so the slopes in the third set are again non-exceptional. Since we know the isolated exceptional fillings on $M_{4}$, to understand those on $M_{5}$ we are only left to understand those on $M_{5}(-2)$, which we can do feeding $M_{5}(-2)$ to our code. But, to avoid considering again the slopes $\infty, 0,1$ that we know to be exceptional on $M_{5}$, and those coming from exceptional slopes on $M_{4}$, we put the preamble

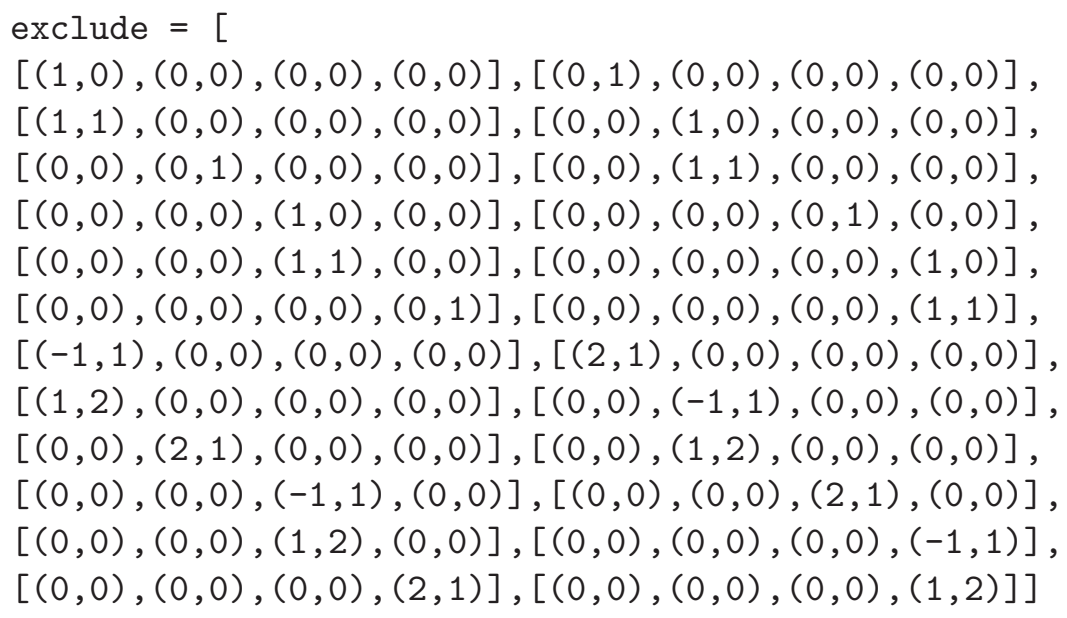

The output of our code is the following:

Candidate exceptional fillings:

With 1 fillings:

[]

Total: 0

With 2 fillings:

[]

Total: 0

With 3 fillings:

[]

Total: 0

With 4 fillings:

$[[(-2,1),(-2,1),(-2,1),(-2,1)],[(-2,1),(1,3),(3,1),(1,3)]$, $[(-1,2),(-2,1),(3,2),(3,2)],[(-1,2),(3,1),(3,1),(-1,2)]$, $[(1,3),(3,1),(1,3),(-2,1)],[(1,3),(3,2),(3,2),(1,3)]$, $[(3,2),(3,2),(-2,1),(-1,2)]]$

Total: 7

Candidate hyperbolic fillings:

With 1 fillings: 
Exceptional Dehn surgery on the minimally twisted five-chain link 725

Table 5: Computer time needed by each code to classify the exceptional fillings of $M_{2}, \ldots, M_{5}$.

\begin{tabular}{lcccc}
\hline & $M_{2}$ & $M_{3}$ & $M_{4}$ & $M_{5}$ \\
\hline find_exceptional_fillings & $1^{\prime \prime}$ & $24^{\prime \prime}$ & $2^{\prime} 10^{\prime \prime}$ & $3^{\prime} 48^{\prime \prime}$ \\
search_geometric_solutions & $<1^{\prime \prime}$ & $<1^{\prime \prime}$ & $<1^{\prime \prime}$ & $<1^{\prime \prime}$ \\
\hline
\end{tabular}

[]

Total: 0

With 2 fillings:

[]

Total: 0

With 3 fillings:

[]

Total: 0

With 4 fillings:

$[[(-2,1),(-2,1),(3,1),(-2,1)],[(-2,1),(1,3),(3,1),(2,3)]$, $[(-2,1),(3,1),(3,1),(-2,1)],[(-1,2),(-2,1),(3,2),(3,1)]$, $[(-1,2),(-1,2),(3,2),(3,2)],[(-1,2),(3,1),(3,1),(-2,1)]$, $[(-1,3),(1,3),(3,2),(2,3)],[(-1,3),(3,1),(-2,1),(-2,1)]$, $[(-1,3),(3,1),(-1,2),(-2,1)],[(1,3),(3,2),(2,3),(2,3)]$, $[(1,3),(4,3),(2,3),(2,3)],[(2,3),(2,3),(3,1),(1,3)]$, $[(3,1),(1,3),(3,1),(1,3)],[(3,2),(3,1),(-1,2),(-2,1)]$, $[(3,2),(3,1),(-1,2),(-1,2)]]$

Total: 15

To achieve tasks (i) and (ii) we then have to show that the 7 candidate exceptional (closed) fillings in list (I) are indeed exceptional, and that the 15 candidate hyperbolic (closed) fillings in list (II) are indeed hyperbolic. For task (i) we take into account the maps (1.1) to (1.3), under which the seven fillings reduce to

$$
(-2,-2,-2,-2,-2) \quad\left(-2,-\frac{1}{2}, 3,3,-\frac{1}{2}\right) .
$$

The Recognizer [25] then confirms that both these fillings are exceptional and give rise to the graph manifolds described in Corollary 1.3. Task (ii) is achieved directly by running the code search_geometric_solutions.py on the 15 candidate hyperbolic manifolds.

To conclude the proof of Theorem 1.2 we are only left to enumerate up to the action of (1.1) to (1.5) the exceptional fillings of $M_{5}$ coming from the 
Table 6: Non-closed isolated exceptional fillings on $M_{4}$, split according to the number $k$ of filled slopes, up to the action of the isometry group of $M_{4}$.

\begin{tabular}{|c|c|c|}
\hline$k$ & Exceptional fillings & Filled manifold \\
\hline \multirow[t]{2}{*}{1} & $(\infty)$ & $P \times S^{1}$ \\
\hline & (0) & $\left(P \times S^{1}\right) \bigcup_{\left(\begin{array}{ll}0 & 1 \\
1 & 0\end{array}\right)}(A,(2,1))$ \\
\hline \multirow[t]{2}{*}{2} & $(-1,-1)$ & $(D,(2,-1),(3,1)) \bigcup_{\left(\begin{array}{ll}0 & 1 \\
1 & 0\end{array}\right)}\left(P \times S^{1}\right)$ \\
\hline & $(-1, \emptyset, 3)$ & $(A,(2,1)) \bigcup_{\left(\begin{array}{ll}0 & 1 \\
1 & 0\end{array}\right)}(A,(2,1))$ \\
\hline \multirow[t]{2}{*}{3} & $(-1,-2,-1) \quad(-2,-1,-2)$ & $\left(P \times S^{1}\right) /\left(\begin{array}{ll}0 & 1 \\
1 & 0\end{array}\right)$ \\
\hline & $\left(-1,-\frac{1}{2}, 4\right) \quad\left(-1, \frac{1}{2}, \frac{5}{2}\right)$ & $(D,(2,-1),(3,1)) \bigcup_{\left(\begin{array}{ll}0 & 1 \\
1 & 0\end{array}\right)}(A,(2,-1))$ \\
\hline
\end{tabular}

exceptional ones on $M_{4}=M_{5}(-1)$ determined in Theorem 3.5. Recall first that

$$
M_{5}\left(-1, \alpha_{2}, \alpha_{3}, \alpha_{4}, \alpha_{5}\right)=M_{4}\left(\alpha_{2}+1, \alpha_{3}, \alpha_{4}, \alpha_{5}+1\right)
$$

and that up to this identification the maps (1.1) to (1.5) generate the maps (3.12) to (3.17) induced by the symmetries of $M_{4}$. So up to (3.12) to (3.17) we see that $\left(-1, \alpha_{2}, \alpha_{3}, \alpha_{4}, \alpha_{5}\right)$ is exceptional for $M_{5}$ if and only if $\left(\alpha_{1}+\right.$ $\left.1, \alpha_{2}, \alpha_{3}, \alpha_{4}+1\right)$ contains one of

$$
\begin{array}{ccc}
0 & \infty & (-1,-2,-1) \\
(-2,-2,-2,-2) & (-1,-3,-2,-3) & (-1,-2,-3,-4)
\end{array}
$$

i.e., if and only if $\left(-1, \alpha_{2}, \alpha_{3}, \alpha_{4}, \alpha_{5}\right)$ contains one of

$$
\begin{array}{ccc}
(-1,-1) & (-1, \infty) & (-1,-2,-2,-1) \\
(-1,-3,-2,-2,-3) & (-1,-2,-3,-2,-4) & (-1,-2,-2,-3,-5)
\end{array}
$$

but we can dismiss $(-1,-1)$ and $(-1, \infty)$ because the latter contains $\infty$, and the former does up to (1.1) to (1.5). The proof is now complete. 
Exceptional Dehn surgery on the minimally twisted five-chain link 727

Table 7: Closed isolated exceptional fillings on $M_{4}$ up to the action of the isometry group of $M_{4}$.

\begin{tabular}{|c|c|}
\hline \multirow{2}{*}{\multicolumn{2}{|c|}{$\begin{array}{l}\text { Exceptional fillings } \\
(-1-2-2-5)\end{array}$}} \\
\hline & \multirow{3}{*}{$\left(S^{2},(3,1),(3,1),(4,-3)\right)$} \\
\hline$(-1,-3,-1,-5)$ & \\
\hline$(-1,-2,-4,-3)$ & \\
\hline$(-1,-2,-2,-4)$ & \multirow{3}{*}{$\left(S^{2},(2,-1),(4,1),(5,1)\right)$} \\
\hline$(-1,-3,-1,-4)$ & \\
\hline$(-1,-2,-3,-3)$ & \\
\hline$(-1,-2,-2,-3)$ & \multirow{2}{*}{$\left(S^{2},(2,-1),(3,1),(7,1)\right)$} \\
\hline$(-1,-3,-1,-3)$ & \\
\hline$(-1,-2,-2,-6)$ & \multirow{3}{*}{$(D,(2,1),(2,-1)) \bigcup_{\left(\begin{array}{cc}-1 & 1 \\
1 & 0\end{array}\right)}(D,(2,1),(3,1))$} \\
\hline$(-1,-3,-1,-6)$ & \\
\hline$(-1,-3,-5,-2)$ & \\
\hline $\begin{array}{c}\left(-1, \frac{1}{2}, \frac{8}{3}, \frac{1}{2}\right) \\
\left(-1,-2,4,-\frac{2}{3}\right)\end{array}$ & $(D,(2,1),(2,-1)) \bigcup_{\left(\begin{array}{ll}1 & 1 \\
0 & -1\end{array}\right)}(D,(2,1),(3,1))$ \\
\hline$\left(-1, \frac{2}{3}, \frac{5}{2}, \frac{2}{3}\right)$ & 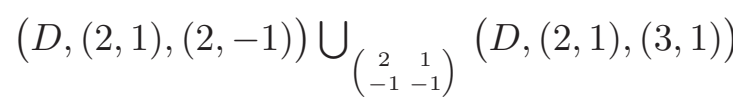 \\
\hline$(-1,-2,-3,-4)$ & \multirow{2}{*}{$(D,(2,1),(2,-1)) \bigcup_{\left(\begin{array}{cc}-1 & 2 \\
1 & -1\end{array}\right)}(D,(2,1),(3,1))$} \\
\hline$(-1,-4,-1,-4)$ & \\
\hline$(-1,-3,-2,-3)$ & $(D,(2,1),(2,-1)) \bigcup_{\left(\begin{array}{cc}-1 & 3 \\
1 & -2\end{array}\right)}(D,(2,1),(3,1))$ \\
\hline$(-2,-2,-2,-2)$ & $(D,(2,1),(2,-1)) \bigcup_{\left(\begin{array}{cc}-1 & 4 \\
1 & -3\end{array}\right)}(D,(2,1),(3,1))$ \\
\hline$\left(-1, \frac{1}{2}, \frac{3}{2}, 3\right)$ & \\
\hline
\end{tabular}


Table 7: Continued.

\begin{tabular}{|c|c|}
\hline$\left(-1,4,5,-\frac{1}{2}\right)$ & $(A,(2,1)) /\left(\begin{array}{ll}0 & 1 \\
1 & 0\end{array}\right)$ \\
\hline$\left(-1,3,4,-\frac{1}{3}\right)$ & $(A,(2,1)) /\left(\begin{array}{ll}1 & 1 \\
1 & 0\end{array}\right)$ \\
\hline$\left(-1, \frac{3}{2}, \frac{5}{2}, \frac{1}{3}\right)$ & $(A,(2,1)) /\left(\begin{array}{ll}2 & 1 \\
1 & 0\end{array}\right)$ \\
\hline
\end{tabular}

Table 8: Non-closed isolated exceptional fillings on $M_{5}$, split according to the number $k$ of filled slopes, up to the action of the isometry group of $M_{5}$.

\begin{tabular}{|c|c|c|}
\hline$k$ & Exceptional fillings & Filled manifold \\
\hline 1 & (1) & $\left(P \times S^{1}\right) \bigcup_{\left(\begin{array}{ll}0 & 1 \\
1 & 0\end{array}\right)}\left(P \times S^{1}\right)$ \\
\hline 2 & $(-1,-1)$ & $\left(P \times S^{1}\right) \cup_{\left(\begin{array}{ll}0 & 1 \\
1 & 0\end{array}\right)}(A,(2,1))$ \\
\hline \multirow[t]{2}{*}{3} & $\begin{array}{l}(-1,-2,-1) \\
(-2,-1,-2)\end{array}$ & $\left(P \times S^{1}\right) \bigcup_{\left(\begin{array}{ll}0 & 1 \\
1 & 0\end{array}\right)}(D,(2,1),(3,1))$ \\
\hline & $\left(\frac{1}{2}, 3, \frac{1}{3}\right)$ & $(A,(2,1)) \bigcup_{\left(\begin{array}{ll}0 & 1 \\
1 & 0\end{array}\right)}(A,(2,1))$ \\
\hline \multirow[t]{2}{*}{4} & $\begin{array}{l}(-1,-2,-2,-1) \\
(-1,-3,-1,-2) \\
(-2,-2,-1,-3)\end{array}$ & $\left(P \times S^{1}\right) /\left(\begin{array}{ll}0 & 1 \\
1 & 0\end{array}\right)$ \\
\hline & $\begin{array}{l}\left(-1,-2, \frac{1}{2}, \frac{5}{2}\right) \\
\left(-2,-1,-\frac{1}{2}, \frac{5}{2}\right) \\
\left(-1,-2,-\frac{1}{2}, 4\right) \\
\left(-2,-1,-\frac{3}{2}, 4\right)\end{array}$ & $(D,(2,-1),(3,1)) \bigcup_{\left(\begin{array}{ll}0 & 1 \\
1 & 0\end{array}\right)}(A,(2,-1))$ \\
\hline
\end{tabular}


Exceptional Dehn surgery on the minimally twisted five-chain link 729

Table 9: Closed isolated exceptional fillings on $M_{5}$ up to the action of the isometry group of $M_{5}$ (part I).

\begin{tabular}{ll}
\hline Exceptional fillings & Filled manifold \\
\hline$(-1,-3,-1,-5,-3)$ & \\
$(-1,-4,-1,-5,-2)$ & \\
$(-1,-2,-2,-2,-6)$ & \\
$(-1,-2,-3,-1,-6)$ & \\
$(-1,-2,-2,-4,-4)$ & \\
$(-1,-2,-5,-2,-3)$ & \\
$(-1,-2,-3,-4,-3)$ & \\
\hline$(-1,-2,-2,-2,-5)$ & \\
$(-1,-2,-3,-1,-5)$ & \\
$(-1,-2,-2,-3,-4)$ & \\
$(-1,-2,-4,-1,-4)$ & \\
$(-1,-3,-1,-4,-3)$ & \\
$(-1,-2,-3,-3,-3)$ & \\
$(-1,-2,-4,-2,-3)$ & \\
\hline$(-1,-2,-2,-2,-4)$ & \\
$(-1,-2,-3,-1,-4)$ & \\
$(-1,-3,-1,-3,-3)$ & \\
$(-1,-2,-1,-3,-2)$ & \\
\hline$(-2,-2,-2,-1,-7)$ \\
$(-1,-2,-3,-1,-7)$ \\
$(-1,-2,-3,-5,-3)$ \\
$(-1,-2,-6,-2,-3)$ \\
$(-1,-3,-1,-6,-3)$ \\
$(-1,-4,-1,-6,-2)$ \\
$(-1,-2,-2,-5,-4)$ \\
\hline
\end{tabular}


Table 9: Continued.

\begin{tabular}{lc}
\hline Exceptional fillings & Filled manifold \\
\hline$\left(-1,-2,-\frac{2}{3}, 4,-3\right)$ & \\
$\left(-1,-2,-2,4,-\frac{5}{3}\right)$ & $(D,(2,1),(2,-1)) \cup_{\left(\begin{array}{ll}1 & 1 \\
0 & -1\end{array}\right)}(D,(2,1),(3,1))$ \\
$\left(-1,-2,3,-1,-\frac{5}{3}\right)$ & \\
$\left(-1,-\frac{1}{2},-1, \frac{1}{2}, \frac{5}{3}\right)$ & \\
\hline
\end{tabular}

\subsection{Computer time}

As thoroughly explained above, during our investigation we have used two different programs: the main python code find_exceptional_fillings and a shorter python code named search_geometric_solutions. Both codes are available from [23], and the computer time spent to run each of them for each manifold $M_{2}, \ldots, M_{5}$ is shown in table 5 . As one can see, once properly organized as we have described, the search requires very little computer time.

\section{Tables}

In this section we expand Theorems 1.2 and 3.5, listing all the isolated exceptional fillings on $M_{4}$ and $M_{5}$, up to the action of their isometry groups. The results stated here are summarized by the entries in table 2 , while those in table 1 were obtained a posteriori using the action of the isometry groups. We also show all the filled manifolds, to do which, in addition to the notation for Seifert and graph manifolds introduced in Section 1.3, we will use $T_{X}$ to denote the torus-bundle on $S^{1}$ obtained from $T \times[0,1]$ by gluing $T \times\{0\}$ to $T \times\{1\}$ along $X \in \mathrm{GL}(2, \mathbb{Z})$ with respect to parallel homology bases; note that for $T_{X}$ to be orientable now one needs to have $\operatorname{det}(X)=+1$.

Theorem 4.1. The isolated exceptional fillings on $M_{4}$, seen up to the action of the isometry group of $M_{4}$ generated by (3.12) to (3.15), are those listed in tables 6 and 7. These fillings are pairwise inequivalent under (3.12) to $(3.15)$. 
Exceptional Dehn surgery on the minimally twisted five-chain link 731

Table 10: Closed isolated exceptional fillings on $M_{5}$ up to the action of the isometry group of $M_{5}$ (part II).

\begin{tabular}{|c|c|}
\hline Exceptional fillings & Filled manifold \\
\hline $\begin{array}{l}\left(-1,-\frac{1}{3},-1, \frac{2}{3}, \frac{3}{2}\right) \\
\left(-1,-\frac{1}{3}, \frac{5}{2}, \frac{2}{3},-2\right)\end{array}$ & $(D,(2,1),(2,-1)) \bigcup_{\left(\begin{array}{cc}2 & 1 \\
-1 & -1\end{array}\right)}(D,(2,1),(3,1))$ \\
\hline $\begin{array}{l}(-1,-3,-1,-4,-4) \\
(-1,-2,-2,-3,-5) \\
(-1,-2,-4,-1,-5) \\
(-1,-2,-4,-3,-3)\end{array}$ & 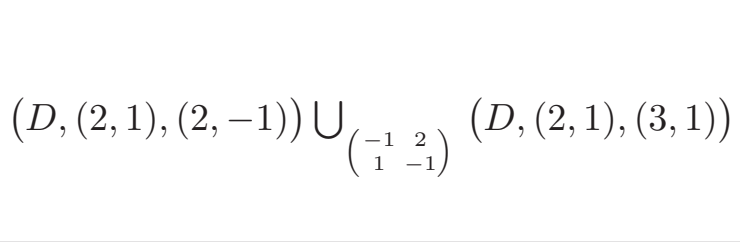 \\
\hline $\begin{array}{l}(-1,-2,-3,-2,-4) \\
(-1,-3,-3,-1,-4)\end{array}$ & $(D,(2,1),(2,-1)) \bigcup_{\left(\begin{array}{cc}-1 & 3 \\
1 & -2\end{array}\right)}(D,(2,1),(3,1))$ \\
\hline$(-1,-3,-2,-2,-3)$ & $(D,(2,1),(2,-1)) \bigcup_{\left(\begin{array}{cc}-1 & 4 \\
1 & -3\end{array}\right)}(D,(2,1),(3,1))$ \\
\hline$(-2,-2,-2,-2,-2)$ & $(D,(2,1),(2,-1)) \bigcup_{\left(\begin{array}{cc}-1 & 5 \\
1 & -4\end{array}\right)}(D,(2,1),(3,1))$ \\
\hline $\begin{array}{l}\left(-1,-2,-1, \frac{1}{2}, \frac{1}{2}\right) \\
\left(-1, \frac{1}{2}, 3,-1,-\frac{1}{2}\right) \\
\left(-2,-1,-\frac{1}{2}, \frac{3}{2}, 3\right)\end{array}$ & $T_{\left(\begin{array}{ll}-3 & 1 \\
-1 & 0\end{array}\right)}$ \\
\hline $\begin{array}{l}\left(-1,-2,-\frac{1}{2}, 5,3\right) \\
\left(-1,-2,4,5,-\frac{3}{2}\right) \\
\left(-1,4,4,-1,-\frac{3}{2}\right)\end{array}$ & $(A,(2,1)) /\left(\begin{array}{ll}0 & 1 \\
1 & 0\end{array}\right)$ \\
\hline
\end{tabular}

(continued) 
Table 10: Continued.

\begin{tabular}{ll}
\hline Exceptional fillings & Filled manifold \\
\hline$\left(-2,-1,2,4,-\frac{1}{3}\right)$ & \\
$\left(-1,-2,3,4,-\frac{4}{3}\right)$ & $(A,(2,1)) /\left(\begin{array}{ll}1 & 1 \\
1 & 0\end{array}\right)$ \\
$\left(-1,-2, \frac{1}{2}, \frac{7}{3}, \frac{1}{3}\right)$ & $(A,(2,1)) /\left(\begin{array}{ll}2 & 1 \\
1 & 0\end{array}\right)$ \\
$\left(-1,-1,-2, \frac{1}{3}, \frac{5}{2}\right)$ & $\left(A, \frac{5}{2},-\frac{2}{3}\right)$ \\
\hline$\left(-1, \frac{1}{2},-1, \frac{1}{3}, \frac{3}{2}\right)$ & $(2,-1)) /\left(\begin{array}{ll}1 & 2 \\
1 & 1\end{array}\right)$ \\
\hline
\end{tabular}

Proof. The discussion in Section 3.5 shows that, up to the action (3.12) to (3.15) of the isometry group of $M_{4}$, an isolated exceptional filling on $M_{4}$ either contains -1 or is equal to $0, \infty$, or $(-2,-2,-2,-2)$. If it contains -1 then it is of type $\left(-1, \alpha_{2}-1, \alpha_{3}, \alpha_{4}-1\right)$ where $\left(\alpha_{2}, \alpha_{3}, \alpha_{4}\right)$ is an isolated exceptional filling on the magic manifold $M_{3}$, as described in Theorem 3.3. But now we are not identifying slopes on $M_{4}$ equivalent under the maps (3.16) and (3.17) induced by isometries of $M_{3}=M_{4}(-1)$ or $M_{2}=M_{4}(-1,-2)$ or $M_{1}=M_{4}(-1,-2,-2)$, therefore we must take the slopes listed in Theorem 3.3, consider their full orbit under the isometries (3.4) and (3.5), pull them back to $M_{4}$, and then remove those that are not isolated on $M_{4}$ and mod out under (3.12) to (3.15). The process is long but straight-forward and leads to the tables, with the manifolds always identified by hand and/or using the Recognizer.

Theorem 4.2. The isolated exceptional fillings on $M_{5}$, seen up to the action of the isometry group of $M_{5}$ generated by (1.1) to (1.3), are those listed in tables 8 to 10. These fillings are pairwise inequivalent under (1.1) to $(1.3)$. 
Exceptional Dehn surgery on the minimally twisted five-chain link 733

Proof. The scheme of the proof is similar, and we omit all details (carried out using a dedicated code). The isolated exceptional fillings on $M_{5}$ described in Theorem 1.2 not coming from $M_{4}$, namely not containing a -1 slope up to (1.1) to (1.3), contribute directly to the tables. Those coming from $M_{4}$ are acted on using the full isometry group of $M_{4}$, pulled back to $M_{5}$, depurated from the non-isolated ones, and modded out under the isometry group of $M_{5}$. Again the manifolds are identified by hand and/or using the Recognizer.

\section{Acknowledgments}

We warmly thank Neil Hoffman and Hidetoshi Masai for many helpful conversations, and the anonymous referees for pointing out a mistake in our tables.

\section{References}

[1] I. Agol, Bounds on exceptional filling, Geom. Topol. 4 (2000), 431-449.

[2] I. Agol, The minimal volume for orientable hyperbolic 2-cusped 3-manifolds, Proc. Amer. Math. Soc. 138 (2010), 3723-3732.

[3] K. Baker, Surgery descriptions and volumes of Berge knots I: large volume Berge knots, J. Knot Theory Ramifications 17 (2008), 1077-1097.

[4] R. Benedetti and C. Petronio, Lectures on hyperbolic geometry, Universitext, Springer-Verlag, New York, 1992.

[5] M. Boileau and J. Porti, Geometrization of 3-orbifolds of cyclic type, Astérisque, 272, 2001, 205 pages.

[6] P.J. Callahan, M.V. Hildebrand and J.R. Weeks, A census of cusped hyperbolic 3-manifolds, Math. Comp. 68 (1999), 321-332.

[7] C. Cao and G.R. Meyerhoff, The orientable cusped hyperbolic 3-manifolds of minimum volume, Invent. Math. 146 (2001), 451-478.

[8] H. Cohen, C. Batut, D. Bernardi, M. Olivier, et al., PARI-GP, http://pari.math.u-bordeaux.fr/

[9] M. Culler, N. Dunfield and J. Weeks, SnapPy, a computer program for studying the geometry and topology of 3-manifolds, http://snappy. computop.org/ 
[10] N. Dunfield and W. Thurston, The virtual Haken conjecture: experiments and examples, Geom. Topol. 7 (2003), 399-441.

[11] D.B.A. Epstein and R.C. Penner, Euclidean decompositions of noncompact hyperbolic manifolds, J. Differential Geom. 27 (1988), 67-80.

[12] M. Eudave-Muñoz, Non-hyperbolic manifolds obtained by Dehn surgery on hyperbolic knots, in 'Geometric topology' (Athens, GA, 1993) AMS/IP Stud. Adv. Math., American Mathematical Society, Providence, RI, 1997, 35-61.

[13] C.McA. Gordon and J. Luecke, Non-integral toroidal Dehn surgeries, Comm. Anal. Geom. 12 (2004), 417-485.

[14] C.McA. Gordon and Y.Q. Wu, Toroidal and annular Dehn fillings, Proc. London Math. Soc. 78 (1999), 662-700.

[15] N. Hoffman, K. Ichihara, M. Kashiwagi, H. Masai, S. Oishi and A. Takayasu, Verified computations for hyperbolic 3-manifolds, arXiv: 1310.3410

[16] N. Hoffman, K. Ichihara, M. Kashiwagi, H. Masai, S. Oishi and A. Takayasu, HIKMOT, a python module of verified computations for hyperbolic 3-manifolds,

www.oishi.info.waseda.ac.jp/ takayasu/hikmot/

[17] K. Ichihara and H. Masai, Exceptional surgeries on alternating knots, arXiv:1310.3472

[18] W. Jaco, P.B. Shalen, A new decomposition theorem for irreducible sufficiently-large 3- manifolds, Algebraic and geometric topology (Proc. Sympos. Pure Math., Stanford Univ., Stanford, Calif., 1976), Part 2, pp. 71-84, Proc. Sympos. Pure Math., XXXII, Amer. Math. Soc., Providence, R.I., 1978.

[19] K. Johannson, Homotopy equivalences of 3-manifolds with boundaries, Lecture Notes in Mathematics, 761. Springer, Berlin, 1979.

[20] K. Jones and A. Reid, Vol 3 and other exceptional hyperbolic 3-manifolds, Proc. Amer. Math. Soc. 129 (2001), 2175-2185.

[21] S. Kang, Reducible and toroidal Dehn fillings with distance 3, Topology 47 (2008), 277-315.

[22] M. Lackenby, Word hyperbolic Dehn surgery, Invent. Math. 140 (2000), 243-282. 
Exceptional Dehn surgery on the minimally twisted five-chain link 735

[23] B. Martelli, Personal web page, www.dm.unipi.it/martelli/Dehn.html

[24] B. Martelli and C. Petronio, Dehn filling of the "magic" 3-manifold, Comm. Anal. Geom. 14 (2006), 969-1026.

[25] S. Matveev and V. Tarkaev, 3-Manifold Recognizer, a computer program for studying the geometry and topology of 3-manifolds, www.matlas.math.csu.ru/

[26] H. Moser, Proving a manifold to be hyperbolic once it has been approximated to be so, Algebr. Geom. Topol. 9 (2009), 103-133.

[27] H. Moser, Personal web page, www.math.columbia.edu/ moser

[28] D. Rolfsen, Knots and links, 2nd edn, Math. Lecture Series, 7, Publish or Perish, Houston, TX, 1990.

[29] W.P. Thurston, The geometry and topology of three-manifolds, Princeton University Mathematics Department, 1979, http://library. msri.org/books/gt3m/.

Dipartimento di Matematica

Largo Pontecorvo 5

56127 PisA, ItALY

E-mail address: martelli@dm.unipi.it

E-mail address: petronio@dm.unipi.it

School of Mathematics and Statistics

UNIVERSITY OF SHEFFIELD

HICKS BUILDING

HOUNSFIELD ROAD

SHEFFIELD, S3 7RH

$\mathrm{UK}$

E-mail address: roukema@gmail.com

ReCEIVED ApRIL 1, 2013 
